\title{
Preliminary study for the laboratory experiment of cosmic-rays driven magnetic field amplification
}

\author{
Chun-Sung Jao ${ }^{\mathrm{a}}$, Sergei Vafina, ${ }^{\mathrm{a}}$, Ye Chen ${ }^{\mathrm{a}}$, Matthias Gross ${ }^{\mathrm{a}}$, Mikhail Krasilnikov ${ }^{\mathrm{a}}$, Gregor Loisch ${ }^{\mathrm{a}}$, Timon Mehrling ${ }^{\mathrm{c}}$, Jacek \\ Niemiec $^{\mathrm{d}}$, Anne Oppelt ${ }^{\mathrm{a}}$, Alberto Martinez de la Ossa ${ }^{\mathrm{e}}$, Jens Osterhoff ${ }^{\mathrm{c}}$, Martin Pohl ${ }^{\mathrm{a}}$, ${ }^{\text {, Frank Stephan }}{ }^{\mathrm{a}}$ \\ ${ }^{a}$ Deutsches Elektronen-Synchrotron DESY, 15738 Zeuthen, Germany \\ ${ }^{b}$ Institute of Physics and Astronomy, University of Potsdam, 14476 Potsdam-Golm, Germany \\ ${ }^{c}$ Deutsches Elektronen-Synchrotron DESY, 22607 Hamburg, Germany \\ ${ }^{d}$ Institute of Nuclear Physics Polish Academy of Sciences, PL-31342 Krakow, Poland \\ ${ }^{e}$ Institute of Experimental Physics, University Hamburg, 22761 Hamburg, Germany
}

\begin{abstract}
To understand astrophysical magnetic-field amplification, we conducted a feasibility study for a laboratory experiment of a nonresonant streaming instability at the Photo Injector Test Facility at DESY, Zeuthen site (PITZ). This non-resonant streaming instability, also known as Bell's instability, is generally regarded as a candidate for the amplification of interstellar magnetic field in the upstream region of supernova-remnant shocks, which is crucial for the efficiency of diffusive shock acceleration. In the beam-plasma system composed of a radio-frequency electron gun and a gas-discharge plasma cell, the goal of our experiment is to demonstrate the development of the non-resonant streaming instability and to find its saturation level in the laboratory environment. Since we find that the electron beam will be significantly decelerated on account of an electrostatic streaming instability, which will decrease the growth rate of desired non-resonant streaming instability, we discuss possible ways to suppress the electrostatic streaming instability by considering the characteristics of a field-emission-based quasi continuous-wave electron beam.
\end{abstract}

Keywords: laboratory astrophysics, beam-plasma instability, magnetic field amplification, radio-frequency electron gun, field-emission-based quasi continuous-wave electron beam

\section{Introduction}

In recent years, many astrophysical laboratories have been established to explore physical processes operating in space ${ }^{25}$ or astrophysical plasmas. With such laboratory experiments, 5 we expand our understanding of physical processes in plasmas and apply it to the extreme astrophysical environments. In this growing field, one of important topics is the generation and amplification of interstellar and intergalactic magnetic fields [1, 2]. ${ }^{30}$ For instance, magnetic-field amplification by plasma turbulence 10 at a laser-produced shock provides experimental evidence for the turbulent amplification of magnetic field in astrophysical environments [3]. In this paper, we propose an experimental investigation of the current-driven magnetic-field amplification, ${ }^{35}$ which is related to the acceleration of high-energy (PeV-scale) 15 cosmic-ray particles.

There is ample observational evidence that high energy cosmic-ray particles are accelerated in supernova remnants, and diffusive shock acceleration is thought as a prime mechanism ${ }^{40}$ for their acceleration $[4,5,6]$. The standard diffusive shock acceleration model indicates that particles would gain energy through multiple shock crossings [7]. To maintain a sufficiently high acceleration rate, turbulent magnetic field much stronger

*Corresponding authors: C.-S. Jao (csjao899@gmail.com), Y. Chen (ye.lining.chen@desy.de), and M.Pohl (martin.pohl@desy.de) than the mean interstellar magnetic field is required to confine particles in the vicinity of the shock $[7,8,9,10]$.

For significant magnetic-field amplification in the upstream region of supernova shocks, a nearly purely growing unstable mode of the non-resonant type has been suggested within the coupled Vlasov-Magnetohydrodynamics (MHD) model [11]. The analytical treatment of this so-called Bell's instability indicated that a saturation level much in excess of the interstellar magnetic-field strength is expected, which could supersede resonant Alfvén-wave amplification [12, 13]. A number of other theoretical studies with MHD or kinetic models has subsequently supported the prospects of the non-resonant streaming instability in magnetic-field amplification $[14,15,16,17$, 18, 19]. High nonlinear saturation levels of the non-resonant streaming instability have been reported in MHD simulations $[11,15,20,21,22]$. However, fully kinetic and hybrid particlein-cell (PIC) models consistently give the saturation amplitudes of only a few times the strength of the homogeneous magnetic field [16, 19, 23, 24, 25, 26, 27, 28]. To examine the saturation level and the saturation mechanism of the non-resonant streaming instability, we attempt to develop a laboratory experiment at the Photo Injector Test (PITZ) facility at DESY, Zeuthen site $[29,30]$. The L-band 1.6-cell normal-conducting (NC) radiofrequency $(\mathrm{RF})$ gun at PITZ is planned to be used as the electron source for our laboratory experiment. The plasma environment in the laboratory for inducing the beam-plasma instabilities is 
expected to be provided by the Gas Discharge Plasma (GDP) PITZ [31]. A variety of advanced beam diagnostics is available downstream of the electron source. To understand the required conditions for the non-resonant streaming instability to occur in our laboratory, a preliminary study is indispensable. We first employ both a standard MHD model and a kinetic model for the linear instability analysis. The PIC simulation model (OSIRIS) $[32,33,34]$ is then used to understand the nonlinear properties of the non-resonant streaming instability.

This paper is organized as follows. A detailed description of the PITZ facility is given in Section 2. The linear instabil-

60 ity analysis, involving typical MHD-based and kinetic models, ${ }_{110}$ is presented in Section 3. Results of preliminary studies with numerical simulations are presented in Section 4. Section 5 contains the summary and discussion.

\section{Overview of the Photo Injector Test Facility at DESY- Zeuthen Site (PITZ)}

The Photo Injector Test facility at DESY, Zeuthen site (PITZ), was built to test, develop and experimentally optimize high brightness photoelectron sources for superconduct- ${ }^{12}$ ing linac driven SASE FELs (SASE: Self-Amplified Spontaneous Emission), such as the Free electron LASer in Hamburg (FLASH) and the European X-ray Free Electron Laser (European XFEL) [35]. The main setup of PITZ consists of an L-band 1.6-cell normal-conducting (NC) RF gun, an L-band ${ }^{125}$ $\mathrm{NC}$ booster cavity, and various advanced systems for cathode 75 laser and electron beam diagnostics, as shown in Fig. 1. Reliable production of high quality electron beams by the PITZ gun along with its advanced beam diagnostics provides excellent opportunities for a wide field of interdisciplinary research activities [36, 37, 38, 39, 40]. More specific introduction of the ${ }^{130}$ gun, the booster cavity and the plasma cell at PITZ are given in the following subsections, respectively.

\subsection{The RF gun}

The key component of the PITZ accelerator is a 1.6-cell RF gun, as shown in Fig. 2. The gun is composed of a copper res85 onator operated at a resonant frequency of $1.3 \mathrm{GHz}$, coaxial RF power coupler, door-knob transition, input waveguide and other ${ }_{140}$ supporting systems. The RF power in the gun is supplied by a 10 MW multi-beam klystron. The power is coupled from the input waveguide via the door-knob transition into the coaxial 90 coupler and the resonator [41]. It allows an accelerating electric field gradient of $60 \mathrm{MV} / \mathrm{m}$ at the position of the cathode. 145 This corresponds to a pulsed peak RF power of about 6.5 MW. A maximum beam momentum of up to $7 \mathrm{MeV} / \mathrm{c}$ at the exit of the gun can be obtained. Moreover, it allows also long pulse operation up to $800 \mu$ s at a repetition rate of $10 \mathrm{~Hz}$ or more. More importantly, operation of the gun has shown a good $\mathrm{RF}_{150}$ amplitude stability of $2 \times 10^{-4}$ and a phase stability of 0.06 degrees.

\subsection{The CDS booster cavity}

Another important accelerator component at PITZ is a NC 14-cell post accelerator booster cavity based on a cut disk structure (CDS, Fig. 3). It was developed by the Institute for Nuclear Research of Russian Academy of Sciences (INR, Moscow) and we put it into operation at PITZ in 2010. It was designed to support long pulse train operation (up to $900 \mu \mathrm{s}$ ) at maximum peak RF power (up to 8.6 MW) [42]. A maximum final beam momentum of $24.5 \mathrm{MeV} / \mathrm{c}$ was obtained when both gun and booster cavities are operated at the phase of the maximum mean momentum gain (MMMG). Besides the functionality of boosting the beam energy, it can also be used as a bunching or debunching cavity for the electron beam by velocity bunching [43].

\subsection{The gas discharge plasma cell}

An Argon gas discharge cell is used at PITZ as a plasma source (see Fig. 4) [31, 38, 39]. The cell consists of two electrodes at the ends of a glass tube which is filled with Argon gas. Polymer foils separate the gas region with pressure of 0.2-8 mbar from the vacuum of the accelerator beam line. The contained Argon gas is ionized and conducts a high current pulse of up to $600 \mathrm{~A}$ for several $\mu$ s when a high voltage is applied between the electrodes. The high current pulse ionizes the gas and heats the plasma. Plasma with densities of up to $5 \times 10^{16} \mathrm{~cm}^{-3}$ is created over a plasma column length of about $10 \mathrm{~cm}$. The plasma density can be varied by changing the delay between plasma ignition and the beam arrival time. For the experiment of the non-resonant streaming instability, a plasma column length up to $45 \mathrm{~cm}$ is needed and Hydrogen or Lithium as a replacement of the Argon gas are considered for the plasma cell.

\subsection{Mechanisms of electron beam generation}

For the experimental investigation of non-resonant streaming instabilities, several quality parameters of the required electron beam are crucial. This includes, e.g. a beam duration on the order of milliseconds, an average beam current of milliamperes, 5 a transverse beam size of roughly $1 \mathrm{~mm}$ in radius, and a beam energy of at least a few $\mathrm{MeV}$. In order to provide suitable electron beams for laboratory experiments, two solutions in terms of generation mechanism are investigated that can utilize the current setup of the PITZ accelerator.

One possible solution is to use field emission from a metallic cathode based on the Fowler-Nordheim theory [44]. Conduction band electrons can quantum mechanically tunnel through the potential barrier at the surface of the metal and be extracted to the vacuum level. This mechanism requires a strong electric field of at least several $\mathrm{GV} / \mathrm{m}$ formed at the so-called field emitter (or needle) cathode. Since the accelerating electric field at the cathode position is about $60 \mathrm{MV} / \mathrm{m}$ in the PITZ gun, the geometry of such a field emitter needs to be specially designed to provide a sufficiently high local field-enhancement factor boosting the electric field to the $\mathrm{GV} / \mathrm{m}$-level. Based on a proper field-emitter design, electron beams of sub-ns FWHM (full-width half maximum) duration with a peak beam current 


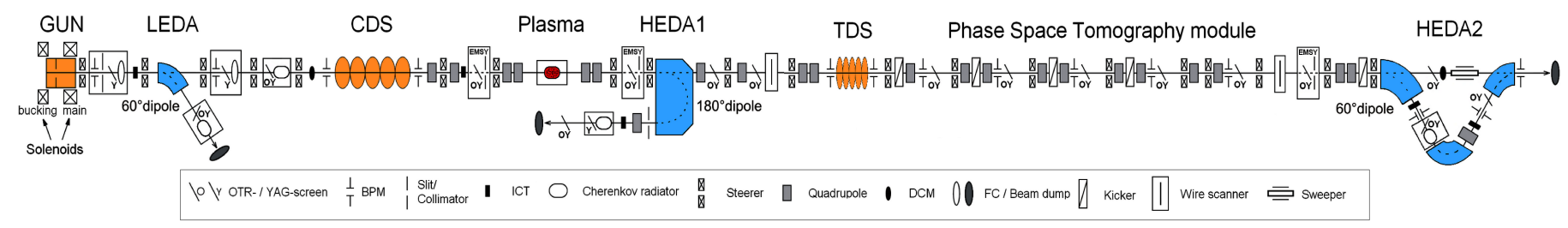

Figure 1: General layout of the PITZ accelerator beamline.
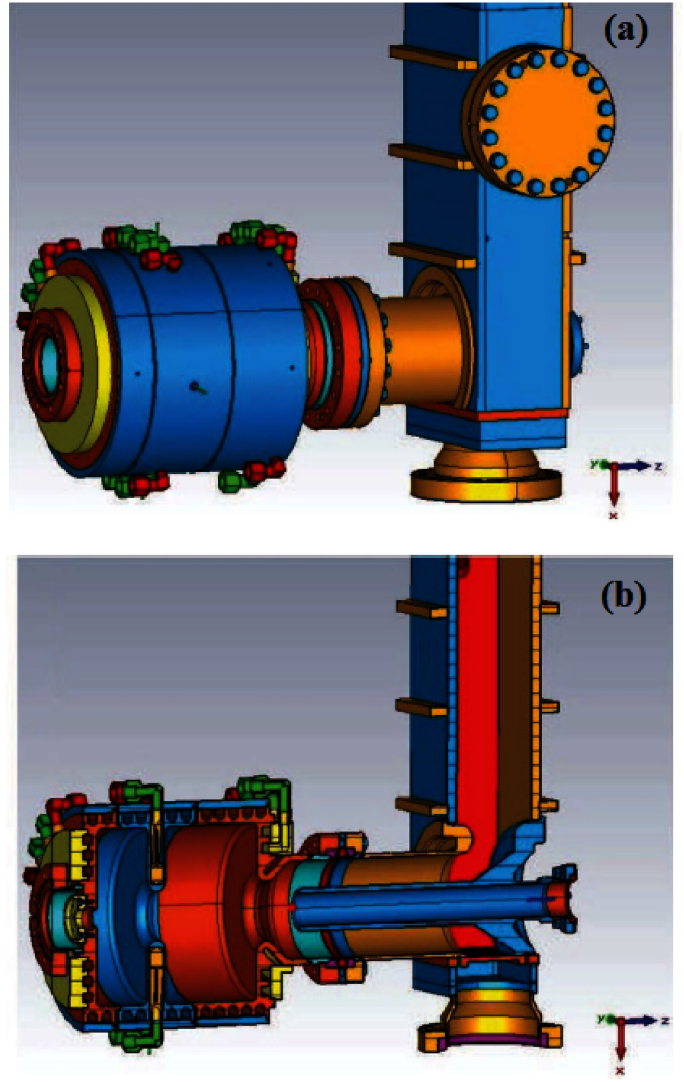

Figure 2: Schematic view of the L-band 1.6-cell PITZ RF gun. (a) Exterior view; (b) Cut-plane View.

of a few tens of mA can be produced. However, the intervals between electron bunches at subsequent RF cycles of the gun are short compared to the RF cycle at $1.3 \mathrm{GHz}$. To join individual bunches from neighboring RF cycles and thus produce a quasi continuous-wave $(\mathrm{CW})$ beam, the booster cavity downstream of the gun can be used for lengthening the bunch duration within each RF period. In such a scheme [40], a quasi-CW electron beam with $\mathrm{mA}$ beam current can be achieved by optimizing the operation conditions.

Another solution to generate the desired electron beam is to use CW-laser-driven photoemission from a high-quantumefficiency (QE) semiconductor photocathode. The choice of a $\mathrm{CW}$ laser overcomes the limitation of the relatively low rep-170 etition rate of the current pulsed laser (at PITZ, $1 \mathrm{MHz}$ ). A high-QE photocathode can emit photoelectrons continuously in
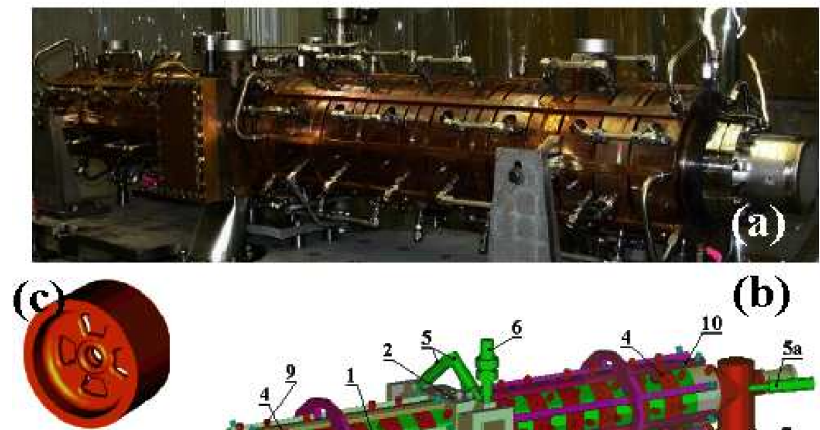

(b)

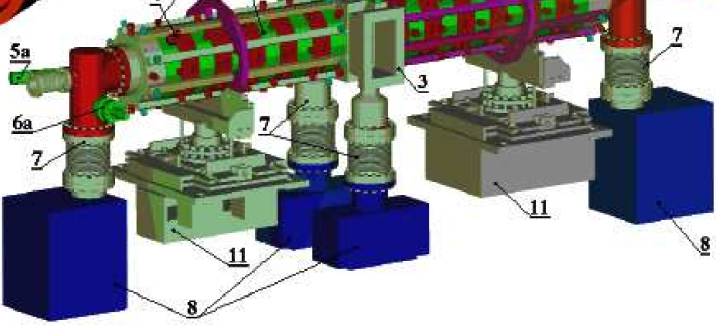

Figure 3: The L-band 14-cell normal conducting PITZ booster cavity.(a) Exterior view of the booster cavity; (b) Sketch of the booster system: 1-regular cells; 2-RF coupler; 3-RF flanges; 5, 5a-photo multipliers; 6, 6a-vacuum gauges; 7pumping ports; 8-ion pumps; 9-internal cooling circuit; 10-outer cooling circuit; 11-support and adjustment.(c) Single cut disk structure.

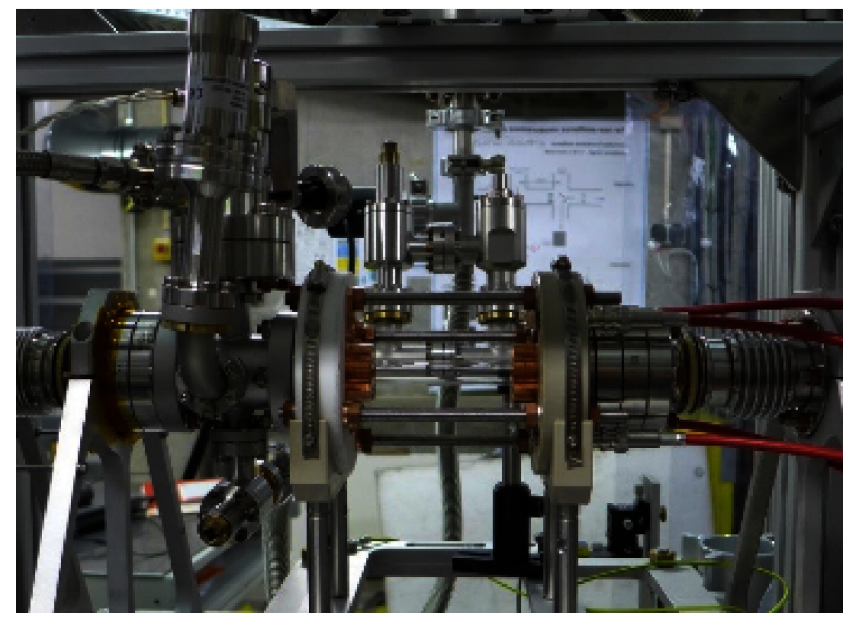

Figure 4: Argon gas discharge plasma (GDP) cell at PITZ [31].The discharge channel is $10 \mathrm{~mm}$ in diameter and $100 \mathrm{~mm}$ in length. A current pulse of 2 to 10 microseconds conducts 200 to 1000 Amperes current.

CW laser operation. The emission of electrons will then depend on the RF fields at a resonance frequency of $1.3 \mathrm{GHz}$. In comparison to the field-emission case, with such a scheme, the temporal profile of a quasi-CW electron beam could be further 
improved in terms of a higher base beam current, due to longer emission time within each RF period. The peak beam current will be increased as well. Systematical beam dynamics studies based on proper photoemission modeling are needed to explore the production and optimization of suitable electron beams in this mechanism.

\subsection{Summary of the laboratory parameters}

In this section we shortly described physical conditions that can be achieved at PITZ to build up a laboratory experiment of beam-plasma instabilities. Based on the laboratory parameters, the linear instability analysis is first performed as a basis for further discussions (Sec. 3). The most basic physical condhe predicted unstable mode wavelength has to be smaller than the size of our plasma cell and that its growth rate has to be large enough for the instability to develop within the plasma lifetime. Laboratory conditions allow the plasma density range from $10^{12}$ to $10^{15} \mathrm{~cm}^{-3}$, and our linear analysis is performed for this range. Because an uninterrupted current is indispensable in the process of the beam-plasma instability development, only the field-emissionbased quasi-CW electron source production process is considered here as the electron source for our experiment. In this case, the available particle density of the electron beam is expected to be in the range $10^{8}$ to $10^{9} \mathrm{~cm}^{-3}$, corresponding to a beam radius of $\sim 1.0 \mathrm{~mm}$. The Lorentz factor in the range 5 to 10 is expected for the quasi $\mathrm{CW}$ electron beam at PITZ. In addition, to neglect the effect of the geomagnetic field on the laboratory experiment, the lowest value of the external magnetic field should be $\sim 1 \mathrm{mT}$, but it can be easily increased up to $0.1 \mathrm{~T}$ in our laboratory. The linear analysis based on these parameters is presented in Section 3.

In theoretical and simulation-based studies of the nonresonant instability a uniform, continuous, and infinite-width beam with relativistic Maxwellian momentum distribution is generally adopted as a cosmic-ray current to induce the beam-225 plasma instability. However, it is not realistic to expect that the electron beam generated in the laboratory would be tallied with 210 such ideal characteristics. An example of the field-emissionbased quasi CW electron beam is shown in Fig. 5. As indicated, due to the limitation in the cycle of the RF gun, the beam electrons can be injected into the plasma cell in term of electron bunches with a repetition rate of $1.3 \mathrm{GHz}$ instead of a continuous beam. In principle, the length of each electron bunch can be extended to the half of an RF cycle, i.e., the equal amount of electrons can be distributed in a bunch with a different length. On the other hand, due to the time-of-flight effect, the momentum distribution in the longitudinal direction is shown with a positive slope from $p_{z}=1.5$ to $5 \mathrm{MeV} / \mathrm{c}$. To understand the effects of a realistic beam in the nonlinear evolution processes, characteristics of the realistic electron beam are also imported in numerical investigations presented in Section 4.
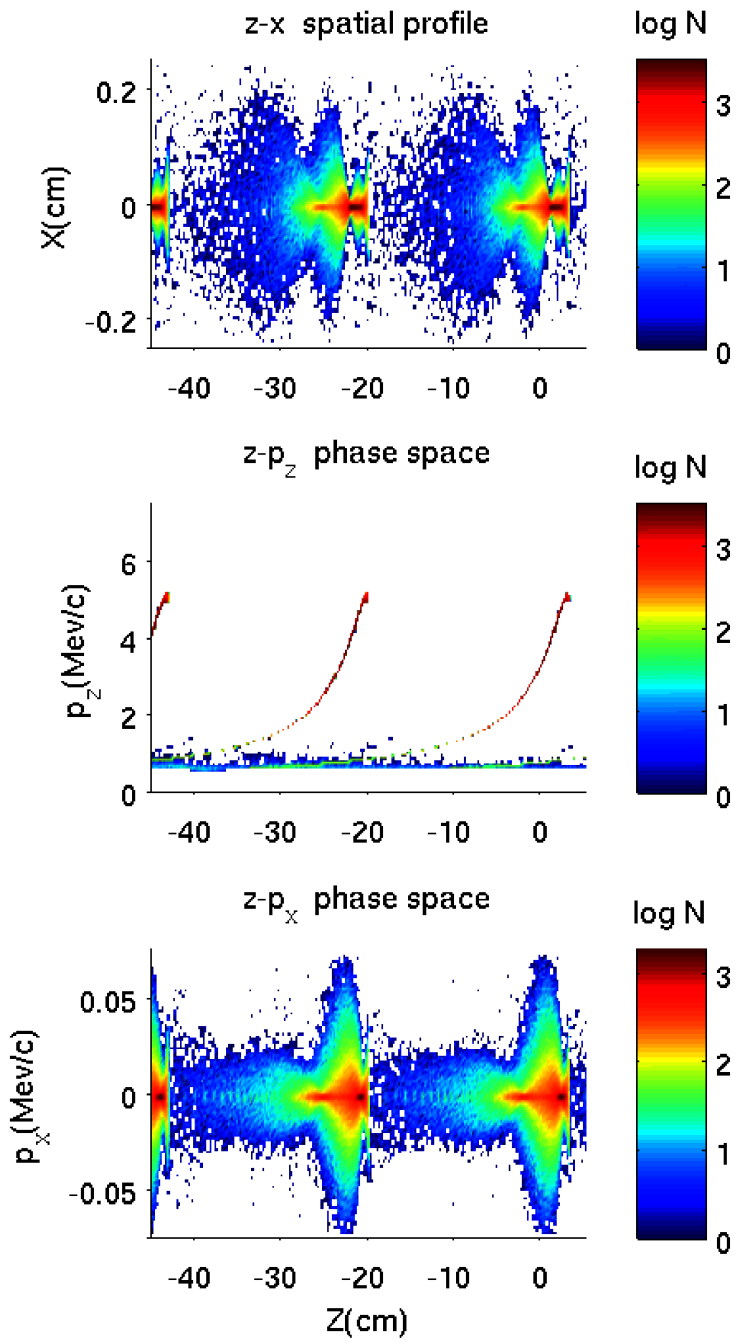

Figure 5: The spatial and momentum distribution of the typical field-emissionbased quasi-CW electron beam based on the 3D particle tracking simulation. The $z$ axis is aligned with the beam.

\section{Linear instability analysis}

\subsection{Non-resonant streaming instability in the MHD model}

For the linear analysis of the non-resonant streaming instability, in the typical Bell's MHD treatment [11], a plasma system with a cosmic-ray current, $\mathbf{j}$, and wavenumber, $\mathbf{k}$, both parallel to the background magnetic field, $\mathbf{B}_{0}\left(\mathbf{j}\|\mathbf{k}\| \mathbf{B}_{0}\right)$ is assumed, where the cosmic-ray current is defined as $\mathbf{j}=n_{b} q \mathbf{v}_{b}\left(n_{b}, q\right.$, and $\mathbf{v}_{b}$ are the particle density, charge, and bulk velocity of cosmicray particles, respectively). The linear treatment indicates that a nearly purely growing non-resonant mode could be excited in the wavenumber regime $1 / r_{L}<k<\mu_{0} j / B_{0}$ with peak growth rate

$$
\gamma_{\max } \simeq \frac{j}{2} \sqrt{\frac{\mu_{0}}{\rho}} \quad \text { at } \quad k_{\max } \simeq \frac{j}{2} \frac{\mu_{0}}{B_{0}} .
$$

Here $\rho=m_{i} n_{0}$ is the density of background plasma $\left(m_{i}\right.$ and $n_{0}$ are the mass of background ions and the particle density of background plasmas, respectively), and the Larmor radius of 
the beam particles, $r_{L}$, is calculated using only the beam speed, $v_{b}$. Significant magnetic-field perturbations can be expected for $k r_{L, b} \gg 1$, which may be expressed as

$$
\frac{\Gamma_{b} \rho_{b} v_{b}^{2}}{2} \frac{\mu_{0}}{B_{0}^{2}} \gg 1
$$

Here $\Gamma_{b}$ is the beam gamma-factor $\Gamma_{b}=\left(1-v_{b}^{2} / c^{2}\right)^{-1 / 2}$. Eq. (2) indicates that for a significant magnetic-field amplification the kinetic energy of the beam must be much larger than the initial ${ }_{260}$ magnetic energy.

Moving from the linear $\left(\delta B / B_{0}<1\right)$ to the non-linear $\left(\delta B / B_{0} \geq 1\right)$ regime, the increasing magnetic tension arising from the magnetic-field amplification will quench further wave growth when

$$
B_{\text {max }} \simeq \frac{\mu_{0} j}{k},
$$

which indicates that modes with small wavenumber could drive stronger magnetic-field perturbations. In other words, unstable modes with larger wavenumber, e.g., $k_{\text {max }}$, would grow quickly but saturate early in the nonlinear processes.

It is to be noted that in the MHD model [11], which is built on the basic MHD assumptions, the growth rate of unstable modes has to be smaller than the ion gyro-frequency. This condition does not necessarily apply to our laboratory experiment, in which an electron beam replaces the cosmic-ray current. Moreover, in the beam-plasma system, numerous instabilities other ${ }_{270}$ than the non-resonant one could be induced in parallel, e.g., the two-stream instability and the O-mode instability [45]. The instability competition must be therefore considered in the nonlinear evolution. This mandates the linear analysis in a kinetic model.

\subsection{Beam-plasma instability in the kinetic model}

In our kinetic model, we consider a beam-plasma system with a relativistic electron beam (with particle density $n_{b}$, speed $v_{b}$, and gamma-factor $\Gamma_{b}$ ) propagating through a fully ionized electron-proton plasma (with the electron and proton densities $n_{e}$ and $n_{p}$, respectively) along a constant magnetic field, $\mathbf{B}_{0}$, i.e., the external magnetic field is parallel to the beam direction. We assume that (i) the beam-plasma system is charge neutral $\left(n_{p}=n_{b}+n_{e}\right)$ and (ii) the beam current is fully balanced by the return current provided by plasma electrons $\left(v_{e}=-\left(n_{b} / n_{e}\right) v_{b} \equiv\right.$ $\left.-\alpha v_{b}\right)$. Additionally, we assume the unperturbed distribution of the beam and plasma is constant and homogeneous. The system of kinetic and Maxwell's equations for such a system can be analytically treated by means of the first-order perturbation analysis resulting in the so-called electromagnetic dispersion equation $[46,47,48,45]$ :

$$
\operatorname{det}\left[\Lambda\left(\omega_{s}(\mathbf{k}), \mathbf{k}\right)\right]=0
$$

where $\mathbf{k}$ denotes the three-component wave vector, $\omega_{s}(\mathbf{k})=$ $\mathfrak{R} \omega_{s}(\mathbf{k})+i \mathfrak{J} \omega_{s}(\mathbf{k})$ is the complex wave frequency, and the ma-285 trix $\Lambda(\omega, \mathbf{k})$ is given in Appendix A. We chose a coordinate system such that the $\mathrm{z}$-axis is directed along the beam (so that components, $\mathbf{k}=\left(k_{\perp}, 0, k_{z}\right)$. A wave corresponding to the solution $\omega_{s}(\mathbf{k})$ is linearly unstable, if the imaginary part of the frequency is positive, $\mathfrak{J} \omega_{s}(\mathbf{k})>0$, for some wave vector $\mathbf{k}$.

For a given k, the dispersion equation (4) has several solutions and, in general, the interplay between individual fluctuation modes is non trivial. Accurate decoupling of different waves is possible under additional assumptions. One possibility is to consider the limiting cases of strictly parallel or perpendicular wave vectors. In the former case, the resonant and nonresonant streaming instabilities can be explored [14, 17], while in the latter case, the behavior of the ordinary (O-)mode instability can be examined [49]. It was already mentioned above that the non-resonant streaming instability (the Bell's mode) can strongly amplify the magnetic field. The same can result from the O-mode instability [50]. Since the focus of our study is magnetic field amplification by the non-resonant streaming instability, it is crucial to investigate conditions for which the O-mode is suppressed compared to the Bell's mode.

Another mode, which can be clearly identified and that is also very important for the beam-plasma interaction, is the electrostatic instability whose dispersion equation can be written as [51]

$$
1-\sum_{a} \frac{m_{a} \omega_{p, a}^{2}}{k^{2}} \int d^{3} p \mathbf{k} \frac{\partial \frac{F_{a}}{\partial} \mathbf{p}}{\mathbf{k}} \mathbf{v}-\omega=0 .
$$

One of the main effects of this instability is the transverse widening of the beam momentum distribution $[52,53]$. This might have a dramatic consequence on the beam coherency by considerably reducing its particle density and, as a result, suppressing the Bell's mode.

In the following subsections, each of the above instabilities is discussed in more details for the beam-plasma parameter range of the PITZ facility presented in Section 2.5.

\subsubsection{Non-resonant streaming instability}

The properties of the non-resonant streaming instability can be explored in the limit of parallel wave vectors. In this case, Eq. (4) becomes [54, 55]:

$$
\begin{aligned}
1-\left(\frac{k c}{\omega}\right)^{2}+\pi \sum_{a} \frac{\omega_{p, a}^{2}}{\omega^{2}} & \int_{-\infty}^{\infty} d p_{z} \int_{0}^{\infty} d p_{\perp} \frac{p_{\perp}^{2}}{\gamma_{a}\left(\omega-k v_{z}\right) \pm \Omega_{a}} \\
\times & {\left[\left(\omega-k v_{z}\right) \frac{\partial f_{a}}{\partial p_{\perp}}+k v_{\perp} \frac{\partial f_{a}}{\partial p_{z}}\right]=0, }
\end{aligned}
$$

where the \pm sign corresponds to the right- $(+)$ and left-handed (-) polarization. For an electron beam, the left-handed mode describes the resonant instability which operates in a narrow wave vector range and, therefore, is problematic for computer simulations due to severe grid-resolution requirements. Therefore, we will focus on the non-resonant mode which is the righthanded wave and which is responsible for magnetic field amplification [11].

The analysis of Eq. (6) requires knowledge of the momentum distribution of the beam and the plasma. We model the background plasma protons with an isothermal and isotropic 
Maxwellian distribution with temperature $T_{p}$,

$$
f_{p}(\mathbf{r}, \mathbf{p})=\frac{n_{p}}{\pi^{3 / 2} p_{p, T}^{3}} \exp \left(-\frac{p^{2}}{p_{p, T}^{2}}\right),
$$

where $p_{p, T}=\sqrt{2 m_{p} T_{p}}$, while for the background electrons we assume

$$
f_{e}(\mathbf{r}, \mathbf{p})=\frac{n_{e}}{\pi^{3 / 2} p_{e, T}^{3}} \exp \left(-\frac{p_{x}^{2}+p_{y}^{2}+\left(p_{z}-p_{e}\right)^{2}}{p_{e, T}^{2}}\right),
$$

where $p_{e, T}=\sqrt{2 m_{e} T_{p}}$. The beam distribution can be relatively well modeled by

$$
f_{b}(\mathbf{r}, \mathbf{p})=\frac{n_{b}}{\pi^{3 / 2} p_{b, \perp}^{2} p_{b, \|}} \exp \left(-\frac{p_{x}^{2}+p_{y}^{2}}{p_{b, \perp}^{2}}-\frac{\left(p_{z}-p_{b}\right)^{2}}{p_{b, \|}^{2}}\right),
$$

where $p_{b, \perp}=\sqrt{2 m_{e} T_{b, R}}, p_{b, \|}=\sqrt{2 m_{e} T_{b, R}} \Gamma_{b}$, and $T_{b, R}$ is the beam temperature in its rest frame.

After inserting Eqs. (7)-(9), Eq. (6) leads to

$$
\begin{gathered}
1-\left(\frac{k c}{\omega}\right)^{2}-\frac{\omega_{p, p}^{2}}{\omega^{2}}\left(1+\frac{1}{2} Z^{\prime}\left(Y_{p}\right)\right)-\frac{\omega_{p, e}^{2}}{\omega^{2}}\left(1+\frac{1}{2} Z^{\prime}\left(Y_{e}\right)\right)- \\
\frac{\omega_{p, b}^{2}}{\Gamma_{b} \omega^{2}}\left(1+\frac{p_{b, \perp}^{2}}{2 p_{b, \|}^{2}} Z^{\prime}\left(Y_{b}\right)\right)-\frac{\Omega_{p} m_{p} \omega_{p, p}^{2}}{k p_{p, T} \omega^{2}} Z\left(Y_{p}\right)- \\
\frac{\Omega_{e} m_{e} \omega_{p, e}^{2}}{k p_{e, T} \omega^{2}} Z\left(Y_{e}\right)-\frac{\Omega_{b} m_{b} \omega_{p, b}^{2}}{k p_{b, \|} \Gamma_{b} \omega^{2}} Z\left(Y_{b}\right)=0,
\end{gathered}
$$

where $Z(x)$ is the plasma dispersion function [56], $Y_{p}=(\omega+$ $\left.\Omega_{p}\right) /\left(k p_{p, T} / m_{p}\right), Y_{e}=\left(\omega+\Omega_{e}-k p_{e} / m_{e}\right) /\left(k p_{e, T} / m_{e}\right), Y_{b}=$ $\left[\omega+\Omega_{b} / \Gamma_{b}-k p_{b} /\left(m_{b} \Gamma_{b}\right)\right] /\left[k p_{b, \|} /\left(m_{b} \Gamma_{b}\right)\right]$. We solve Eq. (10) numerically. For each parameter combination, we search for the maximum of the growth rate $\gamma_{\max }=\max (\mathfrak{J} \omega)$. In case the growth rate has two maxima, we choose the one with a smaller wavelength, since the available plasma length at PITZ is limited. The results are presented in Figs. 6a-6b. To clarify how strongly the beam and plasma temperatures affect the growth rate of the non-resonant streaming instability, in Appendix $\mathrm{C}$ we offer a comparison with the growth rate for a cold beamplasma system. We are especially interested in the right lower corners of Figs. 6 and 16, where the growth rate achieves its maximum and the wave length its minimum. This is crucial since the predicted wavelength has to be smaller than the size of our plasma cell, and the growth rate has to be large enough for Bell's mode to develop within the plasma life time. It is enthe 6 and 16 that the maximum growth rates for the warm and the cold electron beams well agree with each other and approximately equal to $10^{5} \mathrm{~s}^{-1}$. If this instability requires about 20-30 linear growth times to develop, then the plasma life time should be several hundreds microseconds that are available at PITZ. At the same time, the wave length at the maximum growth rate is about $10 \mathrm{~cm}$ and it also demonstrates a rather good agreement for the warm and the cold beams. This wave length can be acceptable for PITZ having the maximum length of the plasma cell of $45 \mathrm{~cm}$. These results provide the most basic physical conditions that permit Bell's instability to occur in our laboratory.

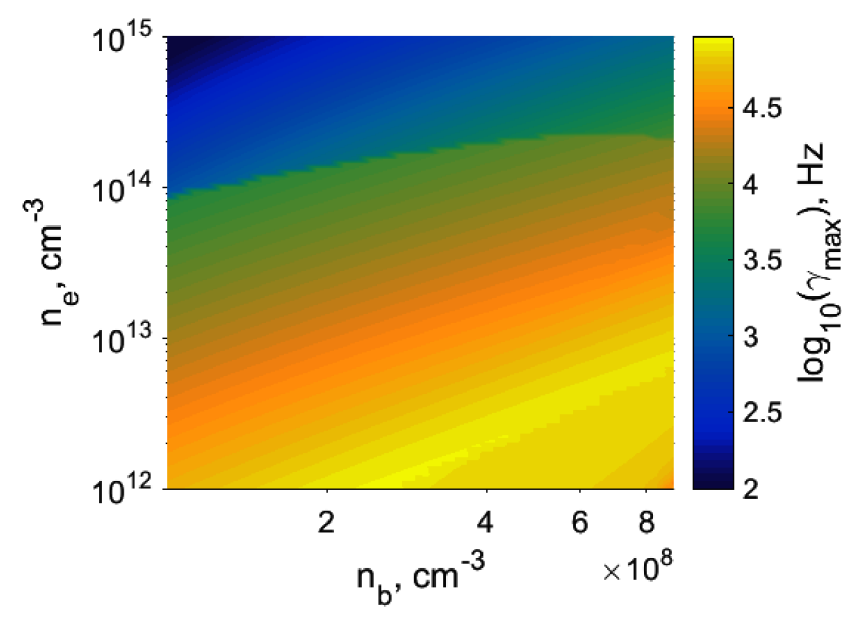

(a)

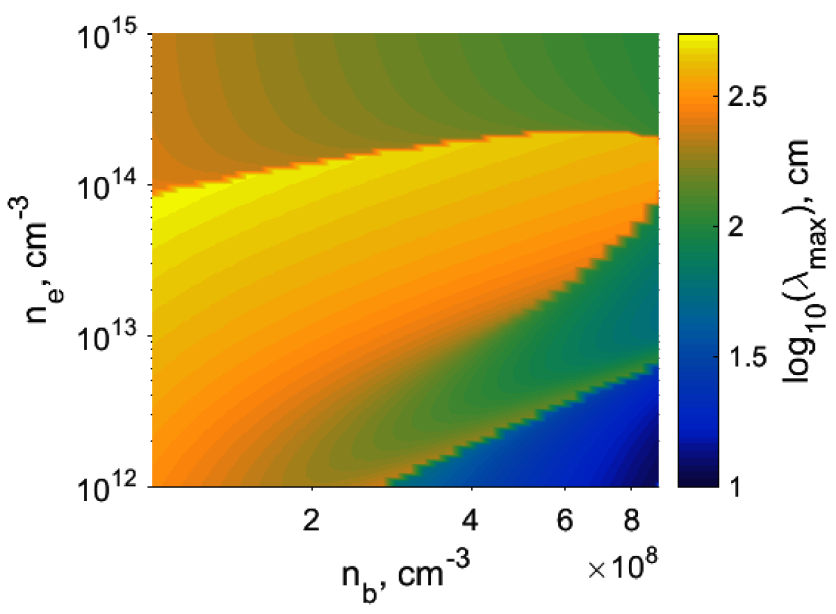

(b)

Figure 6: (a) Maximum growth rate (in units $\mathrm{Hz}$ ) of the non-resonant Bell's instability as function of the beam and plasma density. In case of two maxima, the one at the smaller wavelength is chosen. $B=10^{-3} \mathrm{~T}, \Gamma_{b}=10, T_{b, R}=70$ $\mathrm{eV}, T_{p}=3 \mathrm{eV}$. (b) The wavelength (in units $\mathrm{cm}$ ) of fastest growth of the nonresonant Bell's instability.

\subsubsection{O-mode instability}

For perpendicular wave vectors, $\mathbf{k}=(k, 0,0)$, the dispersion equation (4) reads

$$
\begin{aligned}
\left(\epsilon_{z z}-\left(\frac{k c}{\omega}\right)^{2}\right)\left(\epsilon_{x x}\left(\epsilon_{x x}-\left(\frac{k c}{\omega}\right)^{2}\right)+\epsilon_{x y}^{2}\right)= \\
\epsilon_{x z}^{2}\left(\epsilon_{x x}-\left(\frac{k c}{\omega}\right)^{2}\right)-\epsilon_{x x} \epsilon_{y z}^{2}-2 \epsilon_{x y} \epsilon_{x z} \epsilon_{y z},
\end{aligned}
$$

where the dielectric tensor elements $\epsilon_{i j}$ are given in Appendix $A$ in Eq. (16). The electron beam has a much smaller density than the plasma electrons, $\alpha=n_{b} / n_{e} \ll 1$. It can be easily estimated, using, e.g., the cold beam-plasma limit [51], in which the elements $\epsilon_{x z} \sim \epsilon_{y z} \propto \alpha$. It is clear that the terms on the right-hand side of Eq. (11) are proportional to $\alpha^{2}$, whereas $\epsilon_{x x}$, $\epsilon_{z z}$ and $\epsilon_{x y}$ contain terms proportional only to the first order of $\alpha$. Therefore, we neglect the right-hand side of Eq. (11). Then, 


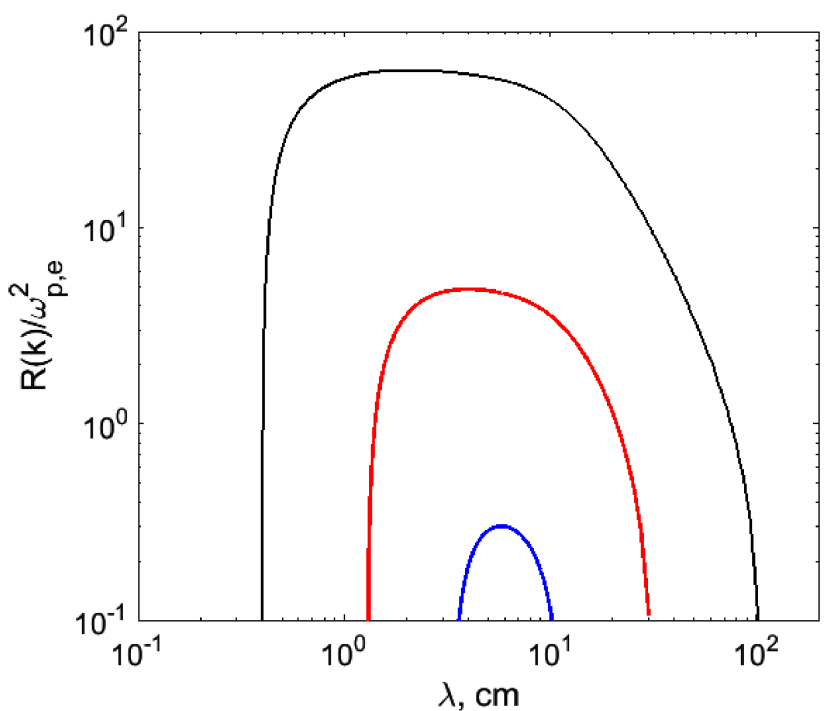

Figure 7: Instability range of the $\mathrm{O}-$ mode as $R(k)>0$ (cf.Eq. (24)) as a function of the wavelength for $n_{e}=10^{12} \mathrm{~cm}^{-3}, T_{b, R}=70 \mathrm{eV}, T_{p}=3 \mathrm{eV}$. Black: $n_{b}=10^{9} \mathrm{~cm}^{-3}$. Red: $n_{b}=10^{8} \mathrm{~cm}^{-3}$. Blue: $n_{b}=10^{7.4} \mathrm{~cm}^{-3}$.

the dispersion equation for the O-mode becomes simply [57]

$$
\epsilon_{z z}-\left(\frac{k c}{\omega}\right)^{2}=0 .
$$

For the distribution functions (7)-(9), we analyze Eq. (12) in Appendix B. It is shown that the O-mode is unstable when $R(k)>0$ (see Eq. (24)). Fig. 7 illustrates $R$ as a function of the wavelength, $\lambda=2 \pi / k$. The O-mode is stable when $R(\lambda)$ is negative, i.e., at small $\lambda$. Thus, the $\mathrm{O}$-mode can be easily stabilized by reducing the beam diameter or the size of the plasma cell to values far below the wavelength range in which the $\mathrm{O}$ mode is unstable. Further discussion is presented in Section 4, in which the simulation results are described.

\subsubsection{Electrostatic instability}

The electrostatic instability was widely investigated in the past $[45,51,58]$. For a hot relativistic beam, the most unstable mode develops in the oblique direction to the beam, and its ${ }_{375}$ growth rate is approximately

$$
\gamma_{\max } \approx 0.4 \Gamma_{b} \frac{n_{b}}{n_{e}} \omega_{p, e} .
$$

\subsection{Summary of linear instability analysis}

In this section, we have presented both the MHD and the kinetic aspects of the linear instability analysis. Since we realized that the O-mode instability will not be induced due to the limited beam diameter and plasma cell size in our system, we only have to consider the electrostatic instability and the non-385 resonant streaming instability in our beam-plasma system. In particular, based on the kinetic model, for $B_{0}=0.1 \mathrm{~T}, \Gamma_{b}=10$, $n_{b}=8 \cdot 10^{8} \mathrm{~cm}^{-3}$, and $n_{e}=10^{13} \mathrm{~cm}^{-3}$, the peak linear growth rate and corresponding wavelength of the electrostatic streaming instability and the non-resonant streaming instability are 390 $\gamma_{\text {max }} \approx 5.7 \cdot 10^{9} \mathrm{~Hz}$ at $1.05 \mathrm{~cm}$ and $\gamma_{\max } \approx 9.5 \cdot 10^{4} \mathrm{~Hz}$ at $16.5 \mathrm{~cm}$, ively, which indicates that the electrostatic streaming instability will be induced much earlier than the Bell's mode. The physical characteristics of the beam and the plasma may be changed by the electrostatic instability with consequences for the subsequent non-resonant streaming instability. The compe5 tition and interaction between these instabilities are investigated numerically and presented in Section 4. According to Bell's MHD treatment, the peak linear growth rate is expected to be $\gamma_{\text {max }} \approx 1.67 \cdot 10^{7} \mathrm{~Hz}$, much higher than the ion gyro-frequency $\omega_{c, i} \approx 9.61 \cdot 10^{5} \mathrm{~Hz}$, which has violated the basic MHD assumpion $\gamma_{\max } \ll \omega_{c, i}$, and so we cannot apply the MHD results for the linear analysis.

\section{Nonlinear numerical simulation}

In this section, the OSIRIS code, a relativistic, massively parallel, and object oriented particle-in-cell (PIC) code [32, , is used for the numerical simulations that bring the system to the nonlinear stage. For saving computational resources, in the following numerical investigation we assume the ion-to-electron mass ratio $m_{i} / m_{e}=50$ and beam density $n_{b}=8.0 \cdot 10^{10} \mathrm{~cm}^{-3}$, which imply the electron skin-depth $c / \omega_{p} \approx 0.17 \mathrm{~cm}$. With these parameters, the linear growth rate and corresponding wavelength of the electrostatic instability are $\gamma_{\text {max }} \approx 5.7 \cdot 10^{9} \mathrm{~Hz}$ at $\lambda \simeq 1.05 \mathrm{~cm}$. As for the non-resonant streaming instability, the kinetic analysis (curve b in Fig. 8) shows an unstable mode appearing in a broad wavelength range, $\lambda \gtrsim 15 \mathrm{~cm}$, with peak growth rate $\gamma_{\max } \approx 7.8 \cdot 10^{7} \mathrm{~Hz}$ at the wavelength $\lambda_{\max } \simeq 26 \mathrm{~cm}$. Comparing the linear analysis results for Lorentz factor $\Gamma_{b}=10$ and mass ratios $m_{i} / m_{e}=1836$ and $m_{i} / m_{e}=50$ (curves (a) and (b) in Fig. 8, respectively), we see that the peak growth rate decreases to $9.5 \cdot 10^{6} \mathrm{~Hz}$ at the realistic mass ratio, but the unstable wavelength range is similar. These result suggests that a longer development time for the non-resonant streaming instability is needed with the real mass ratio, but the expected magnetic turbulence configurations are similar. In Fig. 8 we also show the linear analysis for $m_{i} / m_{e}=50$ and lower Lorentz factor $\Gamma_{b}=5$ (curve (c) in Fig. 8). One can see, that the linear growth rate decreases to $\Gamma_{\max } \approx 5.5 \cdot 10^{7} \mathrm{~Hz}$ in this case.

It is worthy to note that in the MHD limit one finds the peak linear growth rate $\gamma_{\max } \approx 1.05 \cdot 10^{8} \mathrm{~Hz}$ at the wavelength $\lambda_{\max } \approx 26.1 \mathrm{~cm}$, if the mass ratio $m_{i} / m_{e}=50$ is used. Comparing to the ion gyro-frequency, $\omega_{c, i} \approx 3.53 \cdot 10^{8} \mathrm{~Hz}$, we see that the MHD assumption, $\gamma_{\max } / \omega_{c, i}<1$, is satisfied. Bell's criterion for the Larmor radius of beam electrons, $k_{\text {max }}>r_{L, b}^{-1} \simeq 1 / 17 \mathrm{~cm}^{-1}$ is fulfilled as well. If the mass ratio is increased to $m_{i} / m_{e}=1836$, the peak growth rate would drop to $\gamma_{\text {max }} \approx 1.73 \cdot 10^{7} \mathrm{~Hz}$. The peak growth rate predicted with the kinetic model is smaller than that from the MHD treatment, but wavelengths are similar. However, in the MHD limit the predicted peak linear growth and its corresponding wave number (wavelength) are independent of $\Gamma_{b}$ as long as a beam velocity $v_{b} \simeq c$ is assumed. 


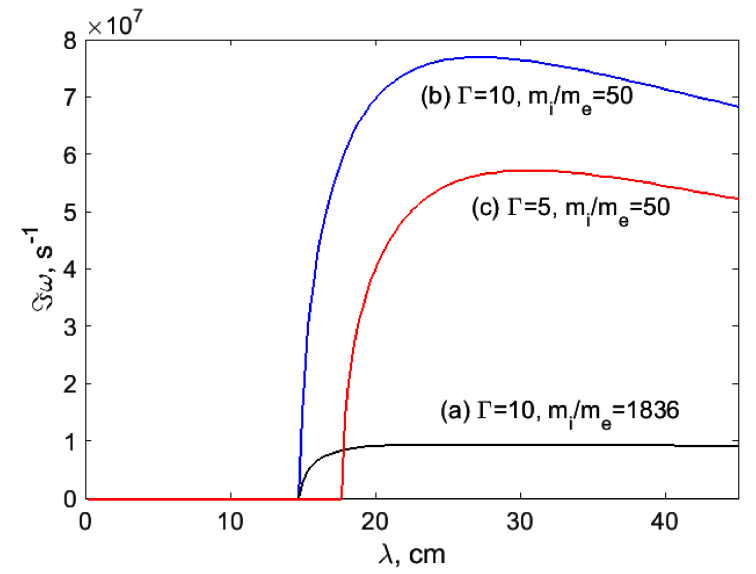

Figure 8: Linear growth rate of Bell's instability for our simulation parameters and (a) $\Gamma_{b}=10$ and $m_{i} / m_{e}=1836$, (b) $\Gamma_{b}=10$ and $m_{i} / m_{e}=50$, and (c) $\Gamma_{b}=5$ and $m_{i} / m_{e}=50$.

\subsection{Electron-beam-induced non-resonant streaming instabil- ity}

In our experiment an electron beam replaces the cosmic-ray current, and so we first employed PIC simulations to explore the electron-beam-induced non-resonant streaming instability. As described in Section 3.3, we assume a stationary plasma with particle density $n_{0}=10^{13} \mathrm{~cm}^{-3}$ within a $72.8 \mathrm{~cm} \times 3.0 \mathrm{~cm}$ $\left(432 c / \omega_{p} \times 18 c / \omega_{p}\right.$ with the cell size $\left.0.125 c / \omega_{p}\right)$ two政 continuous, and infinite-width cold electron beam with relativistic Maxwellian momentum distribution drifting along the $\mathbf{z}$ direction. The particle density and Lorentz factor of the electron beam are set to be $n_{b}=8.0 \times 10^{10} \mathrm{~cm}^{-3}$ and $\Gamma_{b}=10$, $0.1 \mathrm{~T}$, which is also along the $\mathbf{z}$ direction. The background electron component initially drifts in the $-\mathbf{z}$ direction for current neutrality.

The time history of electric and magnetic-field perturbations ${ }^{430}$ . The energy source of beam-plasma instabilities is the bulk energy of the streaming particles, and consequently we observe the beam energy (value of $\Gamma_{b}$ ) decreasing with the electric-field growth at $T \lesssim 1.0 \times 10^{-8} \mathrm{~s}$ and the magnetic-field growth $\mathrm{at}^{435}$

${ }_{415} T \approx 10^{-7} \mathrm{~s}$, when the energy transfer by the electrostatic instability and the non-resonant streaming instability are most efficient.

Fig. 10 shows maps of the magnetic field in the simulation at $T=9.18 \times 10^{-8} \mathrm{~s}\left(16384 \omega_{p}^{-1}, 7.2\right.$ growth time for Bell's mode, ${ }^{440}$ panel (a)) and $1.84 \times 10^{-7} \mathrm{~s}\left(32768 \omega_{p}^{-1}, 14.4\right.$ growth time for Bell's mode, panel (b)). There is no obvious structure in the component along the homogeneous field $\left(B_{z}\right)$. As for the $B_{x}$ and $B_{y}$ components, the magnetic-field structures are coherent and correspond to right-handed circularly polarized plane waves in $^{44}$ the beam direction. Oblique and perpendicular modes such as O-modes are totally suppressed by the limited size of the simulation system in the transverse direction, which is in good agreement with our prediction in Section 3.2.2. We also see that the amplitude of magnetic-field perturbations, $B_{x}$ and $B_{y}$, can grow 450 (a)

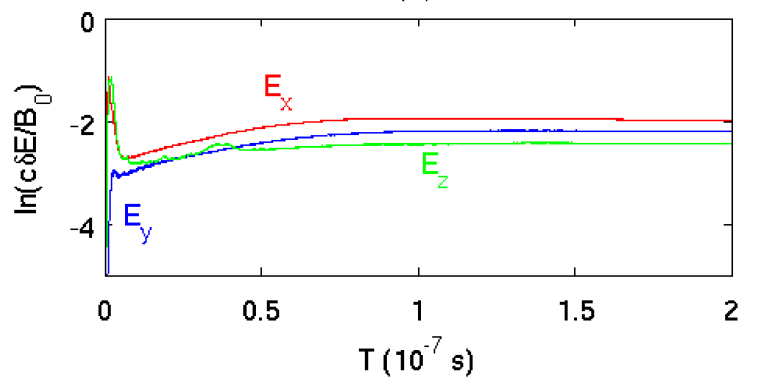

(b)

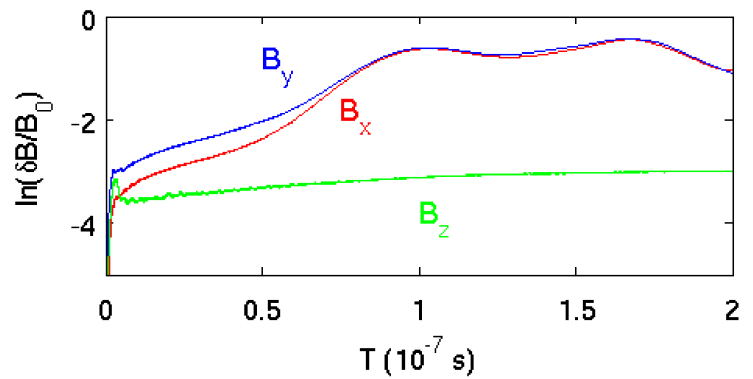

(c)

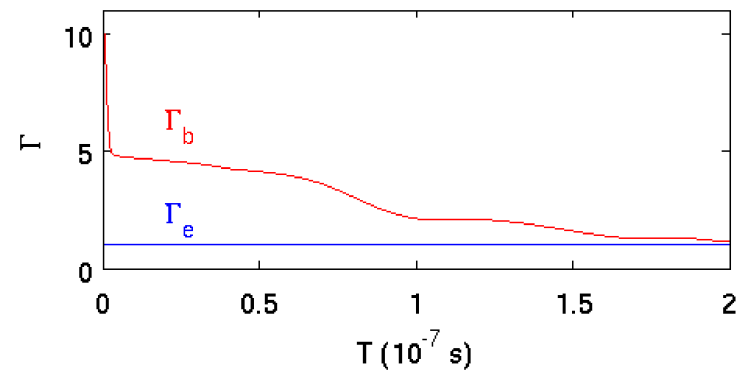

Figure 9: Time evolution of (a) electric-field fluctuations, (b) the magnetic-field amplitude, and (c) the beam Lorentz factor.

to the level of the background magnetic field $(>0.1 T)$, but the wavelength of the dominant field structures changes during the nonlinear evolution. As described in Section 3.1, the most unstable modes with small wavelength will saturate earlier, and the unstable modes with longer wavelength may drive stronger magnetic-field perturbations in the later stage $[11,16,28]$. Figure 11 shows the time evolution of magnetic-field fluctuations in the numerical simulation at different wavelengths. The unstable mode with shortest wavelength, $\lambda=18.20 \mathrm{~cm}$, saturates first, and the unstable mode with wavelength $\lambda=36.40 \mathrm{~cm}$ has the highest saturation level. Here we do not show the wave mode with wavelengths $\lambda \leq 14.56 \mathrm{~cm}$ because they are stable.

Fig. 9 indicates strong reduction of the beam energy in the early stage of the evolution, at $T<1.0 \times 10^{-8} \mathrm{~s}$, which reflects a feedback of the electrostatic instability. Fig. 12 shows maps of the electric field and its corresponding Fourier spectrum at $1.43 \times 10^{-9} s\left(256 \omega_{p}^{-1}\right)$ in the simulation. The electrostatic streaming instability is evidently induced at the expected wavenumber $k \simeq \omega_{\mathrm{pe}} / c \simeq 5.9 \mathrm{~cm}^{-1}(\lambda \simeq 1.05 \mathrm{~cm}$, cf. Section 3.3).

As the beam Lorentz factor drops from $\Gamma_{b}=10$ to $\Gamma_{b}=5$, so 
(a) $T=9.18 \mathrm{e}-08 \mathrm{~s}$

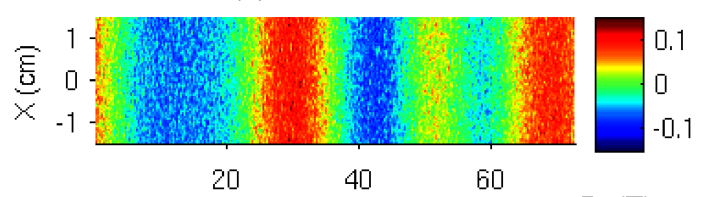

$\mathrm{B}_{y}(\mathrm{~T})$

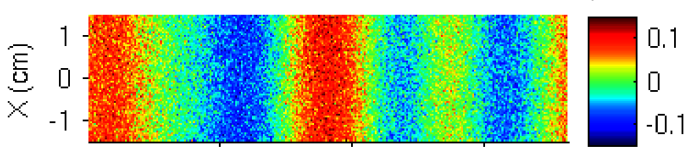

$20 \quad 40 \quad 60$

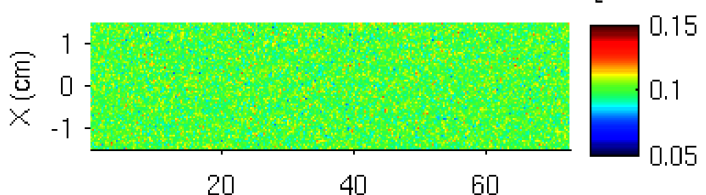

(b) $T=1.84 \mathrm{e}-07 \mathrm{~s}$
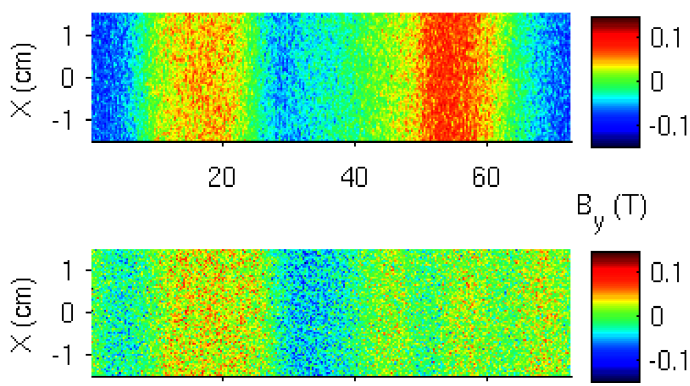

60

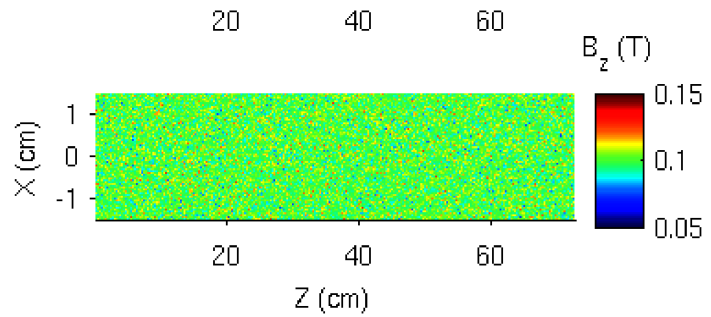

Figure 10: The spatial distribution of the magnetic-field components $B_{x}$ (top panel), $B_{y}$ (middle panel), and $B_{z}$ (bottom panel) at time (a) $T=9.18 \times 10^{-8} s$ $\left(16384 \omega_{p}^{-1}\right)$ and (b) $T=1.84 \times 10^{-7} s\left(32768 \omega_{p}^{-1}\right)$ for the electron-beaminduced Bell's instability. does the growth rate of Bell's mode in our linear analysis (Fig. 8). We can extract from our numerical simulation a growth rate $\sim 6.25 \cdot 10^{7} \mathrm{~Hz}$ for the amplitude of perturbed magnetic field (Fig. 11), as the component at $\lambda=36.40 \mathrm{~cm}$ grows from $\ln \left(\delta B / B_{0}\right)=-6$ to -1 within $\sim 0.8 \times 10^{-7} \mathrm{~s}$. This growth rate is smaller than the linear predictions for $\Gamma_{b}=10$. The slow growth reflects the short development time of the non-resonant streaming instability under the original conditions. During the early evolution the electrostatic instability removed almost half of the beam energy, which decreases the growth rate of the nonresonant streaming instability to the value that is closer to the linear analysis prediction for $\Gamma_{b}=5$. Thus the suppression of the electrostatic instability becomes an important issue. In Section 4.2, we focus on the electrostatic instability for quasi$\mathrm{CW}$ realistic beam characteristics, as opposed to an electron 470

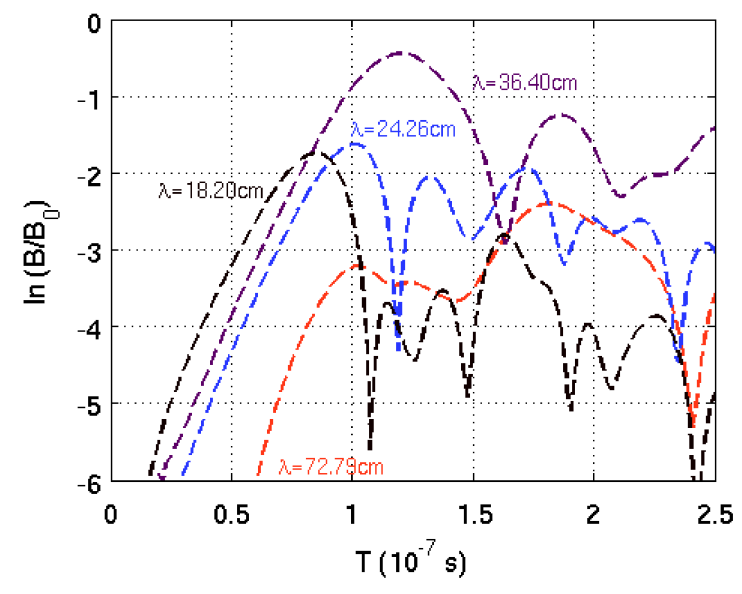

Figure 11: The time evolution of the magnetic-field amplitude at different wavelengths.
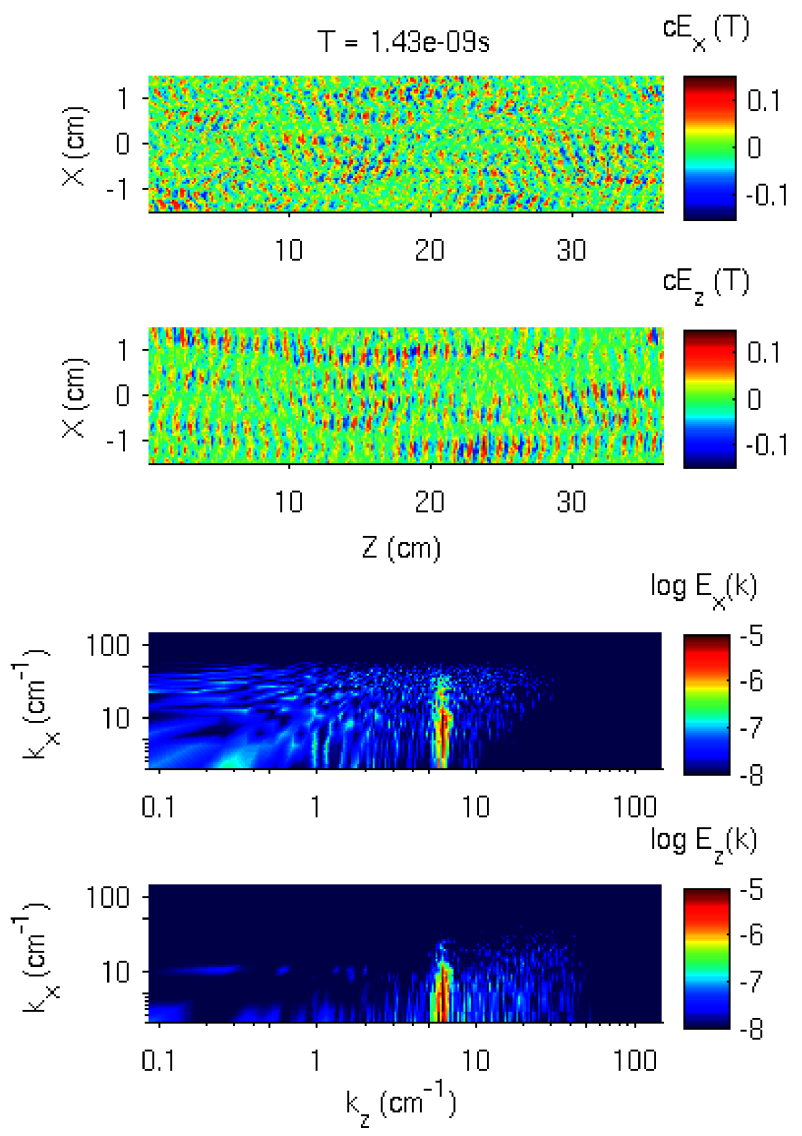

Figure 12: The spatial distribution of the electric-field components $E_{x}$ and $E_{y}$ and the corresponding Fourier spectra at $1.43 \times 10^{-9} s\left(256 \omega_{p}^{-1}\right)$.

beam with ideal characteristics.

\subsection{Electrostatic instability for a non-ideal electron beam}

As the characteristics of a typical field-emission-based quasi$\mathrm{CW}$ electron beam are imported to the simulations, in this section the simulation system is changed from periodic to open 


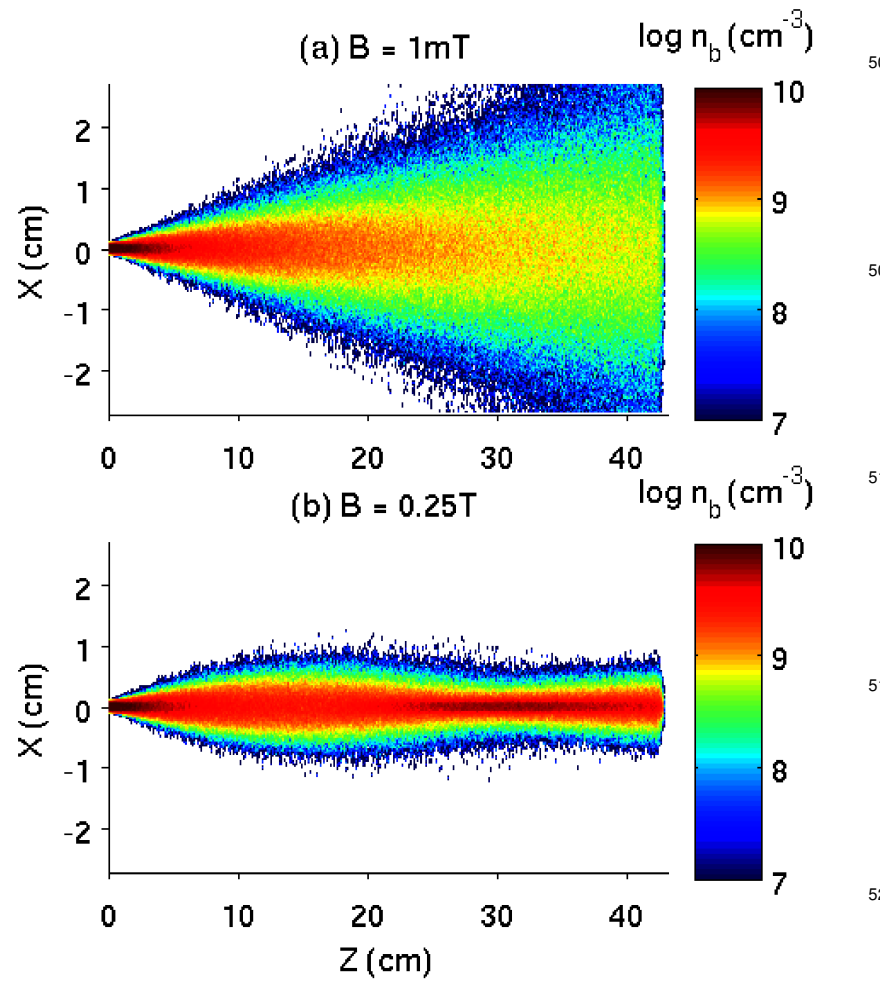

Figure 13: Maps of the logarithmic particle density of electrons in the beam, $\log n_{b}$, for an external magnetic field (a) $1 m T$ and (b) $0.25 T$.

boundary conditions in both $Z$ and $X$ directions. With a stationary plasma with particle density $n_{0}=10^{13} \mathrm{~cm}^{-3}$, the electron beam is injected at $Z=0$ with an initial width $1.7 \mathrm{~mm}$, which is roughly equal to the electron skin length of the background plasma. For any non-zero thermal velocity, the electron beam diverges in the plasma system after being injected. The particle density in the spreading electron beam may quickly decrease, if the thermal velocity is large. Fig. 13(a) indicates that with an isotropic thermal velocity $v_{t h}=0.2 \mathrm{c}$ and an initial width $1.7 \mathrm{~mm}$ of the beam, the beam-core particle density decreases from $n_{b}=10^{10} \mathrm{~cm}^{-3}$ at $Z=0$ to $n_{b}<10^{9} \mathrm{~cm}^{-3}$ at $Z=40 \mathrm{~cm}$. Linear instability analysis predicted the maximum growth rate of the Bell's mode to be directly proportional to beam density $n_{b}$, which implies a non-homogeneous development of the Bell's mode.

To keep the beam core density stable, we may try to minimize the transverse thermal velocity of the electron beam, e.g, by increasing the strength of the external magnetic field, which will ${ }_{545}$ change the gyro-radius and gyro-period of the beam electrons.

490 For instance, if we increase the strength of the external magnetic field from $1 \mathrm{mT}$ (Fig. 13(a)) to $0.25 \mathrm{~T}$ (Fig. 13(b)), the electron beam will only roughly disperse by one thermal gyroradius of the beam electrons (here $0.95 \mathrm{~cm}$ ) and re-converge after half a gyro-period of traveling time (at $29.65 \mathrm{~cm}$ in this case). The particle density of the beam core then keeps being above $n_{b}>10^{9} \mathrm{~cm}^{-3}$. However, the expected wavelength ${ }_{550}$ will also increase with the increasing magnetic field to maintain $k r_{\mathrm{L}}>1$.
As described above, the electrostatic instability in the early 00 decrease the growth rate of the non-resonant streaming instability. To study the suppression of the electrostatic streaming instability with realistic beam characteristics, we consider a noncontinuous electron beam. To mimic the laboratory environment, here we inject an electron bunch every $100 \omega_{p e}^{-1}$, slightly faster than the real RF frequency $1.3 \mathrm{GHz}$. Fig. 14 shows maps of the beam density (top panels), the electric field (middle panels), and the corresponding Fourier spectra (bottom panels) at $T=1.438$ ns $\left(256 \omega_{p e}^{-1}\right)$. We compare results for two lengths of electron bunches, $1 / 4$ of the RF cycle (Fig. 14(a)) and 1/20 RF cycle (Fig. 14(b)). To maintain the same average beam density, the particle density of the electron bunch is 5 times higher in the 1/20-RF-cycle case than it is in the 1/4-RF-cycle case. Electric-field fluctuations arise only along $X=0$ (middle pan515 els), because here we model electron bunches with initial width $1.7 \mathrm{~mm}$ instead of the infinite-width beam underlying Fig. 12.

The Fourier spectra shown in the bottom panels of Fig. 14 indicate that the shorter electron bunches with higher particle density (case 1/20-RF-cycle beam) will induce significantly stronger electric-field perturbations, although the same number of electrons is injected. We conclude that suppression of electrostatic interactions requires stretching of the electron bunches in each RF-cycle.

It is well known that the electrostatic streaming instability can be suppressed by increasing the beam thermal velocity. To test the impact we again use the 1/4-RF-cycle beam but increase the thermal velocity from $v_{t h}=0.01 \mathrm{c}$ (Fig. 15 (a)) to $v_{t h}=$ $0.2 \mathrm{c}$ (Fig. 15(b)). The wider distribution in $p_{z}$ is evident in the top middle panel. The Fourier spectra (bottom panels) of the electric field demonstrate that the electric-field amplitude is indeed substantially weaker for the warm beam (Fig. 15(b)) at the expense of a reduced beam density and hence a lower growth rate of the Bell's instability.

The real beam produced with PITZ hardware is shaped by time-of-flight effects. As shown in Fig. 5, the electron bunches will arrive at the entrance of the plasma cell with a positive slope in the longitudinal momentum distribution. It is important to know whether these electron bunches behave like a warm beam or similar as a cold beam. For numerical investigation we set up another case with the electron bunches mimicking the realistic beam, which is shown in the (c) column of Fig. 15. Note that the length and electron number are unchanged. The Fourier spectra (the bottom panels of Fig. 15) show that the strength of the electric field is similar for the warm beam (Fig. 15(b)) and the realistic beam (Fig. 15(c)), suggesting that the typical field-emission-based quasi-CW electron beam can be approxiamted as a warm beam, which is beneficial for the suppression of the electrostatic streaming instability.

\section{Summary and discussions}

The electromagnetic plasma instability driven by cosmic-ray current, also called Bell's instability, has been discussed as efficient mechanism of the magnetic-field amplification needed for 


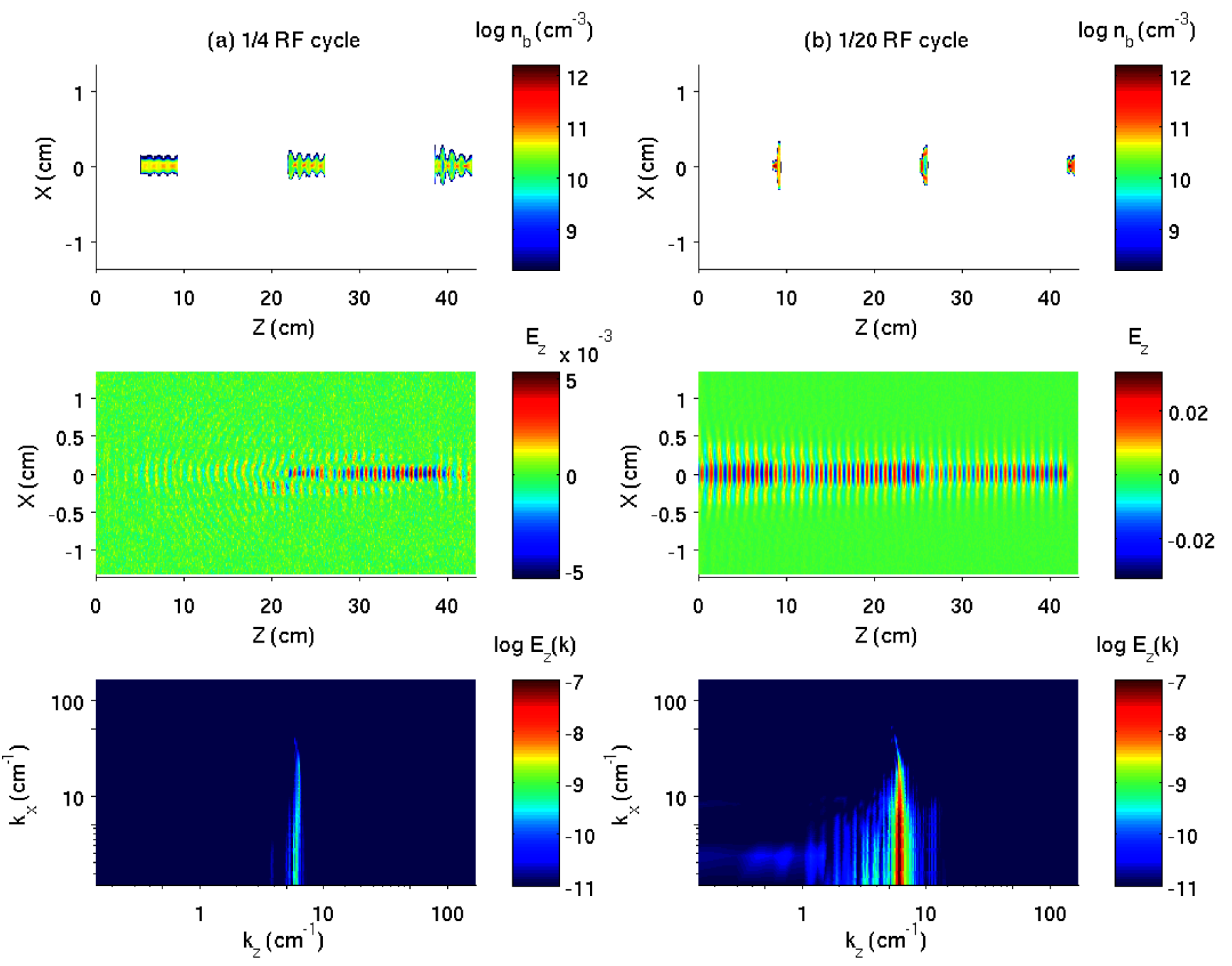

Figure 14: Maps of the electron-beam particle density (top panels), the longitudinal electric field (middle panels, in units $m_{e} c \omega_{p} / e$ ), and the corresponding Fourier spectra (bottom panels) at $T=1.438 \mathrm{~ns}\left(256 \omega_{p e}^{-1}\right.$ ) for electron bunches of length $1 / 4$ (left panels) and $1 / 20$ of the RF period (right panels).

efficient diffusive shock acceleration. To understand its saturation, a laboratory experiment for non-resonant streaming instability is planned at DESY. In this paper we report on preliminary investigations including the facility introduction, linear instability analysis, and nonlinear numerical simulations.

At PITZ, preliminary studies have been performed [40] on the generation and characterization of a quasi-CW electron beam with quality parameters as required for the non-resonant streaming instabilities. We explored two basic mechanisms of electron-beam generation in the PITZ RF gun, including field ${ }^{580}$ emission from a specially designed metallic field emitter as well as CW laser driven photoemission from a high-QE semiconductor photocathode. First simulation results indicated a possibility to produce a quasi-CW electron beam from a specifically designed microtip field emitter sitting on the cathode at 585 the gun-cavity backplane. By using the booster cavity downstream of the gun for bunch lengthening and a few solenoids for beam transverse focusing, a range of beam parameters can be established at the entrance of the plasma cell. This includes a mA-scale average beam current, a few $\mathrm{MeV}$ mean beam energy, and a transverse spot size of about $1 \mathrm{~mm}$ in radius. The impact on the non-resonant streaming instability of the strong modulation in the longitudinal phase space of the electron beam has to be further investigated in the future. Studies on CW-laser driven photoemission-based electron-beam generation are still in progress. Meanwhile, the preparation and possible upgrades of the PITZ plasma cell(s) for fulfilling the requirements of the laboratory experiment are under design considerations.

In our preliminary studies, we use linear analysis to establish the physical conditions that permit the non-resonant streaming instability to occur in the laboratory, the linear growth rate and the corresponding wavelength in particular. Bell's MHD treatment [11] and our kinetic theoretical model are shown to be consistent as long as the parameters fall within the MHD limit. The kinetic model can also work outside of the MHD limit and include temperature effects, and it can in addition provide lin- 

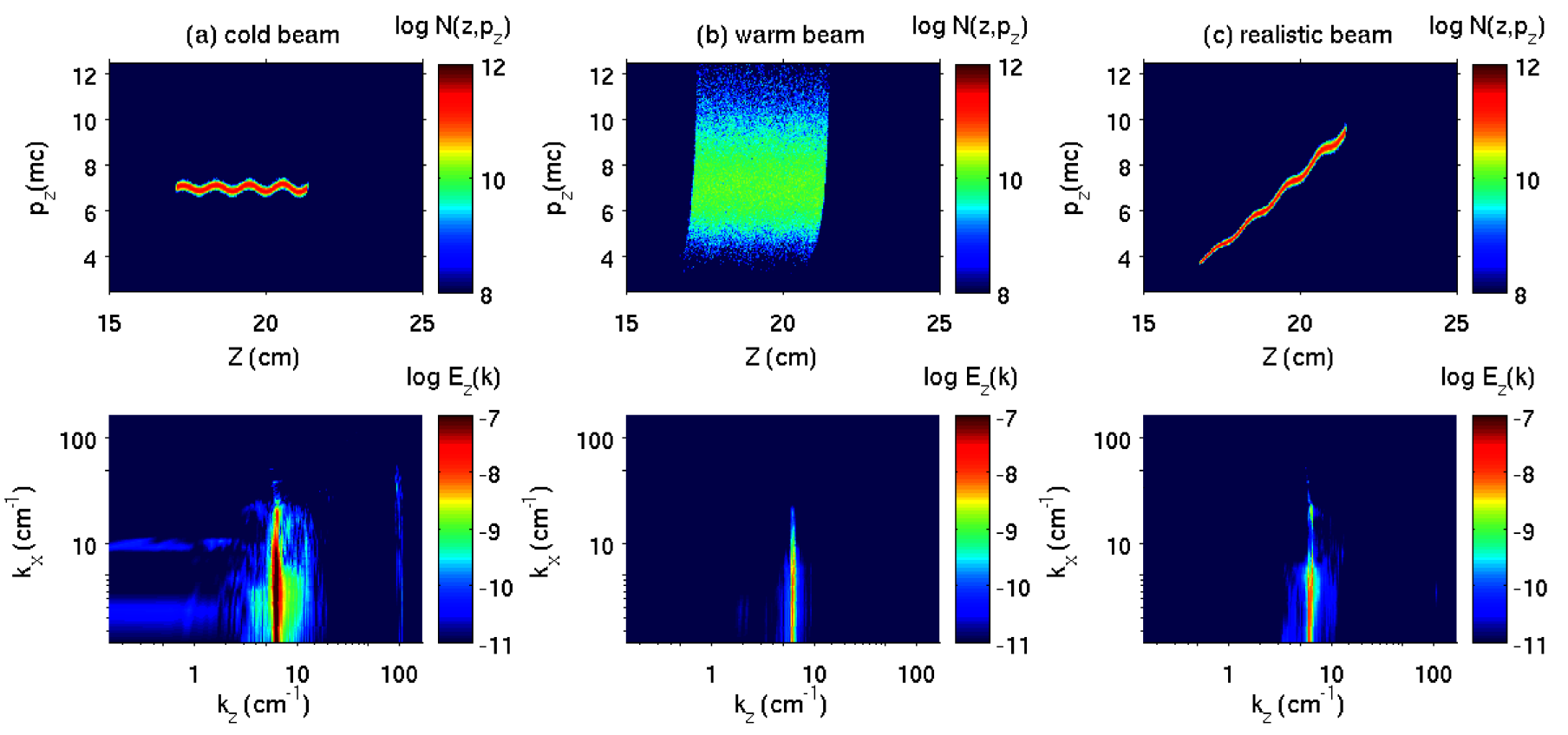

Figure 15: The phase-space distribution of beam electrons (top panels) and the Fourier spectra of the longitudinal electric field (bottom panels) at $\mathrm{T}=5.783$ ns $\left(1024 \omega_{p e}^{-1}\right)$ for (a) cold, (b) warm, and (c) realistic electron bunches.

ear analysis of other instabilities in the beam-plasma system. Finally, PIC simulations are employed to understand the non-620 linear evolution of the non-resonant streaming instability. The simulation results indicate that the electrostatic instability operates in the early stage of evolution processes and can heavily decelerate the electron beam, which will decrease the growth rate of the non-resonant streaming instability.

Thus, the suppression of the electrostatic instability is one of the central issues. For the electron bunches generated in our laboratory, the bunch length may be changed but the amount of injected electrons in each bunch is fixed. We find that shorter bunches with higher particle density induce stronger electricfield perturbations. Our numerical simulations show that the real electron beam with momentum chirps behaves similar to a homogeneous warm beam, as opposed to a cold beam, without showing the beam dispersion of a genuinely warm beam. The electrostatic instability is slowed down further, if longer electrons bunches in each RF-cycle can be established in our experiment. The accompanying head-fast-tail-slow momentum distribution is a benefit for the non-resonant streaming instability to develop in our laboratory setup.

In the end, it can be summarized that the PITZ beam parameters proposed up to now are probably not sufficient experimentally demonstrating with the existing hardware starting the Bell's mode according to our nonlinear calculation. For the currently available beam parameters, the linear growth rate may be too small to be simulated with the PIC technique, which one needs to capture the electrostatic modes. A different numerical model, for instance hybrid simulations, might be considered for further nonlinear studies, and an option might be to use PIC simulation for the early evolution stage and export the beam and plasma data to hybrid simulation for the later evolution. At the same time, we still investigate various possibilities to improve the electron-beam properties based on advanced technologies $[59,60]$. In particular, a higher current density and energy would be beneficial for our laboratory experiment.

\section{Appendix A: matrix $\Lambda(\omega, k)$}

The elements of the matrix $\Lambda$ :

$$
\Lambda=\left(\begin{array}{ccc}
\Lambda_{x x} & \Lambda_{x y} & \Lambda_{x z} \\
\Lambda_{y x} & \Lambda_{y y} & \Lambda_{y z} \\
\Lambda_{z x} & \Lambda_{z y} & \Lambda_{z z}
\end{array}\right)
$$

are determined by $[61,62]$

$$
\Lambda_{i j}=\epsilon_{i j}(\omega, \mathbf{k})-\left(\frac{k c}{\omega}\right)^{2} \delta_{i j}+\frac{k_{i} k_{j} c^{2}}{\omega^{2}}
$$

where $\delta_{i j}$ is the Kronecker symbol and

$$
\epsilon_{i j}=\delta_{i j}-\sum_{a} \frac{m_{a} \omega_{p, a}^{2}}{\omega} \sum_{n=-\infty}^{\infty} \int d^{3} p \frac{S_{i j}}{k_{z} v_{z}-\omega+n \Omega_{a} / \gamma_{a}}
$$

where

$$
S=\left(\begin{array}{ccc}
v_{\perp} U\left(\frac{n J_{n}}{b}\right)^{2} & i v_{\perp} U \frac{n J_{n} J_{n}^{\prime}}{b} & v_{\perp} \frac{n J_{n}^{2}}{b}\left(\frac{n W}{b}+\frac{\partial F_{a}}{\partial p_{z}}\right) \\
-i v_{\perp} U \frac{n J_{n} J_{n}^{\prime}}{b} & v_{\perp} U J_{n}^{\prime 2} & -i v_{\perp} J_{n} J_{n}^{\prime}\left(\frac{n W}{b}+\frac{\partial F_{a}}{\partial p_{z}}\right) \\
v_{z} U \frac{n J_{n}^{2}}{b} & i v_{z} U J_{n} J_{n}^{\prime} & v_{z} J_{n}^{2}\left(\frac{n W}{b}+\frac{\partial F_{a}}{\partial p_{z}}\right)
\end{array}\right),
$$


where

$$
\begin{gathered}
U=\left(1-\frac{k_{z} v_{z}}{\omega}\right) \frac{\partial F_{a}}{\partial p_{\perp}}+\frac{k_{z} v_{\perp}}{\omega} \frac{\partial F_{a}}{\partial p_{z}}, \\
W=\frac{k_{\perp} v_{z}}{\omega} \frac{\partial F_{a}}{\partial p_{\perp}}-\frac{k_{\perp} v_{\perp}}{\omega} \frac{\partial F_{a}}{\partial p_{z}},
\end{gathered}
$$

$J_{n}=J_{n}\left(k_{\perp} v_{\perp} \gamma_{a} / \Omega_{a}\right)$ is the Bessel function of the first kind, $F_{a}(\mathbf{p})=f_{a}(\mathbf{r}, \mathbf{p}) / n_{a}$ is the momentum distribution of species $a$ with the particle density $n_{a}, \omega_{p, a}=\left(4 \pi n_{a} e_{a}^{2} / m_{a}\right)^{1 / 2}, \Omega_{a}=$ $e_{a} B_{0} /\left(m_{a} c\right), \gamma_{a}=\left(1+p^{2} /\left(m_{a} c\right)^{2}\right)^{1 / 2}$.

\section{Appendix B: dispersion equation of O-mode}

For the distribution functions (7)-(9), it can be easily proved that Eq. (11) has a purely real or a purely imaginary solution $[49,57]$. For a purely imaginary solution $(\omega=i \mathfrak{J} \omega)$, Eq. (11) can be written as $[57,63]$

$$
(\mathfrak{J} \omega)^{2}=\frac{R(k)}{L(k, \mathfrak{J} \omega)},
$$

where

$$
\begin{aligned}
& L(k, \mathfrak{J} \omega)=1- \\
& 2 \sum_{a} m_{a} \omega_{p, a}^{2} \int d^{3} p \frac{v_{z}^{2}}{v_{\perp}} \frac{\partial F_{a}}{\partial p_{\perp}} \sum_{n=1}^{\infty} \frac{\left[J_{n}\left(k v_{\perp} \gamma_{a} / \Omega_{a}\right)\right]^{2}}{(\mathfrak{J} \omega)^{2}+\left(n \Omega_{a} / \gamma_{a}\right)^{2}} \\
& R(k)=-(k c)^{2}+\sum_{a} m_{a} \omega_{p, a}^{2} \int d^{3} p v_{z} \frac{\partial F_{a}}{\partial p_{z}}- \\
& \sum_{a} m_{a} \omega_{p, a}^{2} \int d^{3} p \frac{v_{z}^{2}}{v_{\perp}} \frac{\partial F_{a}}{\partial p_{\perp}}\left(1-\left[J_{0}\left(k v_{\perp} \gamma_{a} / \Omega_{a}\right)\right]^{2}\right) .
\end{aligned}
$$

Since $L(k, \mathfrak{J} \omega)>0$, Eq. (20) has real solutions only for $R(k) \geq$ 0 , thus, yielding the instability condition of the O-mode. For the distributions (7)-(9) and approximating $\gamma_{e} \approx 1, \gamma_{b} \approx \Gamma_{b}$, we obtain

$$
\begin{gathered}
R(k)=-(k c)^{2}-\omega_{p, p}^{2}-\omega_{p, e}^{2}-\frac{\omega_{p, b}^{2}}{\Gamma_{b}^{3}}+ \\
2 \frac{\omega_{p, b}^{2}}{\Gamma_{b}} \frac{p_{b}^{2}}{p_{b, \perp}^{2}} W\left(x_{b}\right)+\omega_{p, e}^{2} W\left(x_{e}\right)\left(1+2 \frac{p_{e}^{2}}{p_{e, T}^{2}}\right)+\omega_{p, p}^{2} W\left(x_{p}\right),
\end{gathered}
$$

where $x_{b}=\left[k p_{b, \perp} /\left(m_{e} \Omega_{e}\right)\right]^{2} / 2, x_{e}=\left[k p_{e, T} /\left(m_{e} \Omega_{e}\right)\right]^{2} / 2, x_{p}=$ $\left[k p_{p, T} /\left(m_{p} \Omega_{p}\right)\right]^{2} / 2$, and

$$
W(x)=1-\exp (-x) I_{0}(x) .
$$

Here, $I_{0}$ denotes the modified Bessel function of the first kind.

\section{Appendix C: non-resonant Bell's instability for a cold beam- plasma system}

In the cold beam-plasma approximation, the distribution functions of the beam, background electrons and ions become, respectively,

$$
f_{b}\left(p_{\perp}, p_{z}\right)=n_{b} \frac{\delta\left(p_{\perp}\right)}{2 \pi p_{\perp}} \delta\left(p_{z}-p_{b}\right),
$$

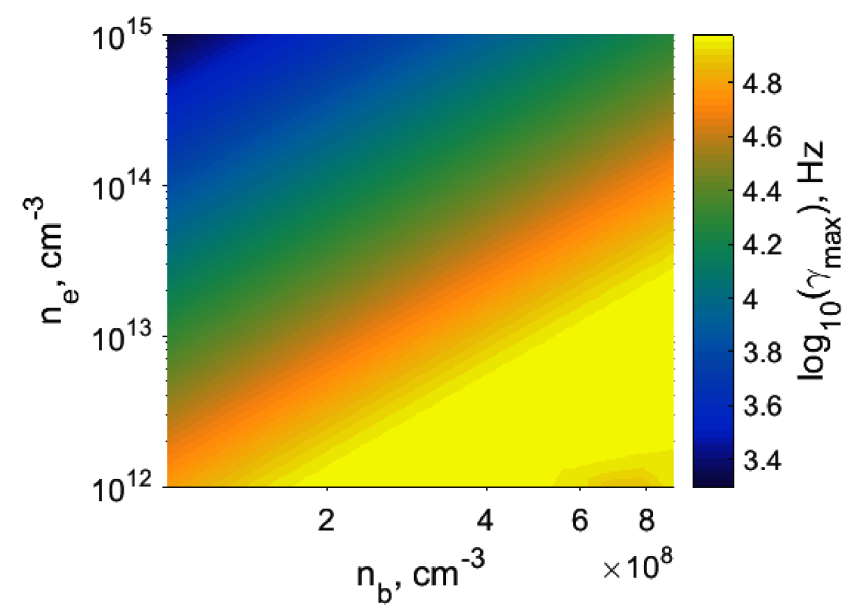

(a)

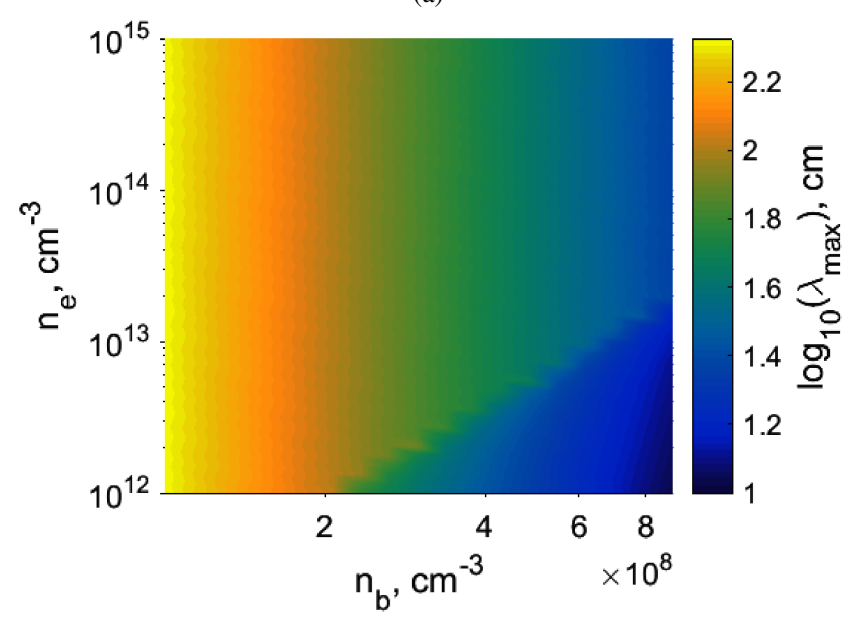

(b)

Figure 16: Same as Fig. 6, but for the cold beam-plasma system.

$$
\begin{gathered}
f_{e}\left(p_{\perp}, p_{z}\right)=n_{e} \frac{\delta\left(p_{\perp}\right)}{2 \pi p_{\perp}} \delta\left(p_{z}-p_{e}\right), \\
f_{i}\left(p_{\perp}, p_{z}\right)=n_{i} \frac{\delta\left(p_{\perp}\right)}{2 \pi p_{\perp}} \delta\left(p_{z}\right) .
\end{gathered}
$$

After inserting Eqs. (25)-(27) into Eq. (6), we derive the well35 known dispersion equation [51]

$$
\begin{array}{r}
1-\left(\frac{k c}{\omega}\right)^{2}-\frac{\omega_{p, p}^{2}}{\omega\left(\omega \pm \Omega_{p}\right)}-\frac{\omega_{p, b}^{2}}{\omega^{2}} \frac{\omega-k V_{b}}{\Gamma_{b}\left(\omega-k V_{b} \pm \Omega_{b} / \Gamma_{b}\right)}- \\
-\frac{\omega_{p, e}^{2}}{\omega^{2}} \frac{\omega-k V_{e}}{\Gamma_{e}\left(\omega-k V_{e} \pm \Omega_{e} / \Gamma_{e}\right)}=0
\end{array}
$$

where the upper sign corresponds to the non-resonant Bell's mode. The maximum growth rate and the corresponding wave number for the same beam-plasma parameters as Fig. 6 are demonstrated in Fig. 16. 


\section{Acknowledgements}

This research is supported by the Deutsches ElektronenSynchrotron (DESY) Strategy Fund. The computational resources for the numerical simulations are provided by DESY and the Norddeutsche Verbund zur Förderung des Hoch- ${ }^{710}$ und Höchstleistungsrechnens (HLRN) under project bbp00015. The work of $\mathrm{J}$. Niemiec is supported by Narodowe Centrum Nauki through research project DEC-2013/10/E/ST9/00662. C.-S. Jao also thanks for the kind feedback from K. Makwana.

\section{References}

[1] G. Gregori, B. Reville, F. Miniati, The generation and amplification of intergalactic magnetic fields in analogue laboratory experiments with high power lasers, Physics Reports 601 (2015) 1 - 34.

[2] Y. Kuramitsu, Y. Sakawa, T. Morita, T. Ide, K. Nishio, H. Tanji, H. Aoki, S. Dono, C. D. Gregory, J. N. Waugh, N. Woolsey, A. Dizire, A. Pelka, A. Ravasio, B. Loupias, M. Koenig, S. A. Pikuz, Y. T. Li, Y. Zhang, X. Liu, J. Y. Zhong, J. Zhang, G. Gregori, N. Nakanii, K. Kondo, Y. Mori, E. Miura, R. Kodama, Y. Kitagawa, K. Mima, K. A. Tanaka, H. Azechi, T. Moritaka, Y. Matsumoto, T. Sano, A. Mizuta, N. Ohnishi, ${ }_{730}$ M. Hoshino, H. Takabe, Laboratory investigations on the origins of cosmic rays, Plasma Physics and Controlled Fusion 54 (2012) 124049.

[3] J. Meinecke, H. W. Doyle, F. Miniati, A. R. Bell, R. Bingham, R. Crowston, R. P. Drake, M. Fatenejad, M. Koenig, Y. Kuramitsu, C. C. Kuranz, D. Q. Lamb, D. Lee, M. J. MacDonald, C. D. Murphy, H.-S. Park, ${ }_{735}$ A. Pelka, A. Ravasio, Y. Sakawa, A. A. Schekochihin, A. Scopatz, P. Tzeferacos, W. C. Wan, N. C. Woolsey, R. Yurchak, B. Reville, G. Gregori, Turbulent amplification of magnetic fields in laboratory laser-produced shock waves, Nature Physics 10 (2014) 520.

[4] A. R. Bell, The acceleration of cosmic rays in shock fronts i, Monthly ${ }_{740}$ Notices of the Royal Astronomical Society 182 (1978) 147-156.

5] J. P. Blandford, R. D.; Ostriker, Particle acceleration by astrophysical shocks, The Astrophysical Journal 221 (1978) L29.

[6] L. O. Drury, An introduction to the theory of diffusive shock acceleration of energetic particles in tenuous plasmas, Reports on Progress in Physics ${ }_{745}$ 46 (1983) 973.

[7] K. M. Schure, A. R. Bell, L. O'C Drury, A. M. Bykov, Diffusive shock acceleration and magnetic field amplification, Space Science Reviews 173 (2012) 491-519.

[8] M. A. Malkov, P. H. Diamond, Modern theory of fermi acceleration: $A_{750}$ new challenge to plasma physics, Physics of Plasmas 8 (2001) 24012406.

[9] J. Vink, J. M. Laming, On the magnetic fields and particle acceleration in cassiopeia a, The Astrophysical Journal 584 (2003) 758.

[10] H. J. Völk, E. G. Berezhko, L. T. Ksenofontov, Magnetic field amplification in tycho and other shell-type supernova remnants, Astronomy \& Astrophysics 433 (2005) 229-240.

[11] A. R. Bell, Turbulent amplification of magnetic field and diffusive shock acceleration of cosmic rays, Monthly Notices of the Royal Astronomical Society 353 (2004) 550-558.

[12] R. Kulsrud, W. P. Pearce, The effect of wave-particle interactions on the propagation of cosmic rays, The Astrophysical Journal 156 (1969) 445.

[13] J. F. McKenzie, H. J. Voelk, Non-linear theory of cosmic ray shocks including self-generated alfven waves, The Astrophysical Journal 116 (1982) 191

[14] B. Reville, J. G. Kirk, P. Duffy, A current-driven instability in parallel, rel- ${ }^{765}$ ativistic shocks, Plasma Physics and Controlled Fusion 348 (2006) 1741

[15] V. N. Zirakashvili, V. S. Ptuskin, H. J. Völk, Modeling bell's nonresonant cosmic-ray instability, The Astrophysical Journal 678 (2008) 255.

[16] J. Niemiec, M. Pohl, T. Stroman, K.-I. Nishikawa, Production of Mag- ${ }_{770}$ netic Turbulence by Cosmic Rays Drifting Upstream of Supernova Remnant Shocks, The Astrophysical Journal 684 (2008) 1174-1189.

[17] E. Amato, P. Blasi, A kinetic approach to cosmic-ray-induced streaming instability at supernova shocks, Monthly Notices of the Royal Astronomical Society 392 (2009) 1591.
[18] Q. Luo, D. Melrose, Saturated magnetic field amplification at supernova shocks, Monthly Notices of the Royal Astronomical Society 397 (2009) 1402-1409.

[19] M. A. Riquelme, A. Spitkovsky, Nonlinear study of bell's cosmic ray current-driven instability, The Astrophysical Journal 694 (2009) 626.

[20] V. N. Zirakashvili, V. S. Ptuskin, Diffusive shock acceleration with magnetic amplification by nonresonant streaming instability in supernova remnants, The Astrophysical Journal 678 (2008) 939.

[21] F. Casse, A. Marcowith, R. Keppens, Non-resonant magnetohydrodynamics streaming instability near magnetized relativistic shocks, Monthly Notices of the Royal Astronomical Society 433 (2013) 940-951.

[22] J. H. Matthews, A. R. Bell, K. M. Blundell, A. T. Araudo, Amplification of perpendicular and parallel magnetic fields by cosmic ray currents, Monthly Notices of the Royal Astronomical Society 469 (2017) 1849 1860 .

[23] Y. Ohira, B. Reville, J. G. Kirk, F. Takahara, Two-dimensional particlein-cell simulations of the nonresonant, cosmic-ray-driven instability in supernova remnant shocks, The Astrophysical Journal 698 (2009) 445.

[24] T. Stroman, M. Pohl, J. Niemiec, Kinetic simulations of turbulent magnetic-field growth by streaming cosmic rays, The Astrophysical Journal 706 (2009) 38

[25] L. Gargat, R. A. Fonseca, J. Niemiec, M. Pohl, R. Bingham, L. O. Silva, The nonlinear saturation of the non-resonant kinetically driven streaming instability, The Astrophysical Journal Letters 711 (2010) L127.

[26] J. Niemiec, M. Pohl, A. Bret, T. Stroman, Aperiodic magnetic turbulence produced by relativistic ion beams, The Astrophysical Journal 709 (2010) 1148 .

[27] M. A. Riquelme, A. Spitkovsky, Magnetic amplification by magnetized cosmic rays in supernova remnant shocks, The Astrophysical Journal 717 (2010) 1054.

[28] O. Kobzar, J. Niemiec, M. Pohl, A. Bohdan, Spatio-temporal evolution of the non-resonant instability in shock precursors of young supernova remnants, Monthly Notices of the Royal Astronomical Society 469 (2017) 4985-4998

[29] F. Stephan, C. H. Boulware, M. Krasilnikov, J. Bähr, G. Asova, A. Donat, U. Gensch, H. J. Grabosch, M. Hänel, L. Hakobyan, H. Henschel, Y. Ivanisenko, L. Jachmann, S. Khodyachykh, M. Khojoyan, W. Köhler, S. Korepanov, G. Koss, A. Kretzschmann, H. Leich, H. Lüdecke, A. Meissner, A. Oppelt, B. Petrosyan, M. Pohl, S. Riemann, S. Rimjaem, M. Sachwitz, B. Schöneich, T. Scholz, H. Schulze, J. Schultze, U. Schwendicke, A. Shapovalov, R. Spesyvtsev, L. Staykov, F. Tonisch, T. Walter, S. Weisse, R. Wenndorff, M. Winde, L. v. Vu, H. Dürr, T. Kamps, D. Richter, M. Sperling, R. Ovsyannikov, A. Vollmer, J. Knobloch, E. Jaeschke, J. Boster, R. Brinkmann, S. Choroba, K. Flechsenhar, K. Flöttmann, W. Gerdau, V. Katalev, W. Koprek, S. Lederer, C. Martens, P. Pucyk, S. Schreiber, S. Simrock, E. Vogel, V. Vogel, K. Rosbach, I. Bonev, I. Tsakov, P. Michelato, L. Monaco, C. Pagani, D. Sertore, T. Garvey, I. Will, I. Templin, W. Sandner, W. Ackermann, E. Arévalo, E. Gjonaj, W. F. O. Müller, S. Schnepp, T. Weiland, F. Wolfheimer, J. Rönsch, J. Rossbach, Detailed characterization of electron sources yielding first demonstration of european $\mathrm{X}$-ray free-electron laser beam quality, Physical Review Accelerators and Beams 13 (2010) 020704.

[30] M. Krasilnikov, F. Stephan, G. Asova, H.-J. Grabosch, M. Groß, L. Hakobyan, I. Isaev, Y. Ivanisenko, L. Jachmann, M. Khojoyan, G. Klemz, W. Köhler, M. Mahgoub, D. Malyutin, M. Nozdrin, A. Oppelt, M. Otevrel, B. Petrosyan, S. Rimjaem, A. Shapovalov, G. Vashchenko, S. Weidinger, R. Wenndorff, K. Flöttmann, M. Hoffmann, S. Lederer, H. Schlarb, S. Schreiber, I. Templin, I. Will, V. Paramonov, D. Richter, Experimentally minimized beam emittance from an $l$-band photoinjector, Physical Review Accelerators and Beams 15 (2012) 100701.

[31] G. Loisch, et al., Experimental Investigation of High Transformer Ratio Plasma Wakefield Acceleration at PITZ, in: Proceedings of 8th International Particle Accelerator Conference (IPAC 2017), 2017, p. TUPIK018.

[32] R. A. Fonseca, L. O. Silva, F. S. Tsung, V. K. Decyk, W. Lu, C. Ren, W. B. Mori, S. Deng, S. Lee, T. Katsouleas, J. C. Adam, Osiris: A threedimensional, fully relativistic particle in cell code for modeling plasma based accelerators, in: P. M. A. Sloot, A. G. Hoekstra, C. J. K. Tan, J. J. Dongarra (Eds.), Computational Science - ICCS 2002: International Conference Amsterdam, The Netherlands, April 21-24, 2002 Proceedings, Part III, 2002, pp. 342-351 
[33] R. A. Fonseca, S. F. Martins, L. O. Silva, J. W. Tonge, F. S. Tsung, W. B. Mori, One-to-one direct modeling of experiments and astrophysical scenarios: pushing the envelope on kinetic plasma simulations, Plasma Physics and Controlled Fusion 50 (2008) 124034.

[34] R. A. Fonseca, J. Vieira, F. Fiuza, A. Davidson, F. S. Tsung, W. B. Mori,850 L. O. Silva, Exploiting multi-scale parallelism for large scale numerical modelling of laser wakefield accelerators, Plasma Physics and Controlled Fusion 55 (2013) 124011

[35] F. Stephan, M. Krasilnikov, High Brightness Photo Injectors for Brilliant Light Sources, in: E. Jaeschke, S. Khan, J. R. Schneider, J. B. Hastings855 (Eds.), Synchrotron Light Sources and Free-Electron Lasers: Accelerator Physics, Instrumentation and Science Applications, Springer International Publishing Switzerland, 2014, pp. 1 - 38.

[36] P. Boonpornprasert, G. Asova, Y. Chen, J. Good, H. Huck, I. Isaev, et al., Experimental Optimization and Characterization of Electron Beams for 860 Generating IR/THz SASE FEL Radiation with PITZ, in: Proceedings of 8th International Particle Accelerator Conference (IPAC 2017), International Particle Accelerator Conference, JACoW, Geneva, Switzerland, 2017, pp. 2650-2653.

[37] F. Lemery, P. Piot, G. Amatuni, P. Boonpornprasert, Y. Chen, J. Good,865 B. Grigoryan, M. Groß, M. Krasilinikov, O. Lishilin, G. Loisch, A. Oppelt, S. Philipp, H. Qian, Y. Renier, F. Stephan, I. Zagorodnov, Passive ballistic microbunching of nonultrarelativistic electron bunches using electromagnetic wakefields in dielectric-lined waveguides, Physical Review Letters 122 (2019) 044801.

[38] M. Gross, O. Lishilin, G. Loisch, P. Boonpornprasert, Y. Chen, J. Engel, J. Good, H. Huck, I. Isaev, M. Krasilnikov, X. Li, R. Niemczyk, A. Oppelt, H. Qian, Y. Renier, F. Stephan, Q. Zhao, R. Brinkmann, A. M. de la Ossa, J. Osterhoff, F. J. Grner, T. Mehrling, C. B. Schroeder, I. Will, Characterization of self-modulated electron bunches in an argon plasma,875 Journal of Physics: Conference Series 1067 (2018) 042012.

[39] G. Loisch, G. Asova, P. Boonpornprasert, R. Brinkmann, Y. Chen, J. Engel, J. Good, M. Gross, F. Grüner, H. Huck, D. Kalantaryan, M. Krasilnikov, O. Lishilin, A. M. de la Ossa, T. J. Mehrling, D. Melkumyan, A. Oppelt, J. Osterhoff, H. Qian, Y. Renier, F. Stephan, C. Tenholt,880 V. Wohlfarth, Q. Zhao, Observation of high transformer ratio plasma wakefield acceleration, Physical Review Letters 121 (2018) 064801.

[40] Y. Chen, G. Loisch, M. Gross, C.-S. Jao, M. Krasilnikov, A. Oppelt, et al., Generation of quasi continuous-wave electron beams in an 1-band normal conducting pulsed rf injector for laboratory astrophysics experiments, $\mathrm{Nu}-885$ clear Instruments and Methods in Physics Research, A 903 (2018) 119 125.

[41] Y. Renier, et al., Statistics on High Average Power Operation and Results from the Electron Beam Characterization at PITZ, in: Proceedings of 8th International Particle Accelerator Conference (IPAC 2017), International Particle Accelerator Conference, JACoW, Geneva, Switzerland, 2017, pp. 1806-1808.

[42] V. Paramonov, et al., The PITZ CDS Booster Cavity RF Tuning and Start of Conditioning, in: Proceedings, 25th International Linear Accelerator Conference, LINAC2010, 2011, p. MOP081.

[43] L. Serafini, M. Ferrario, Velocity bunching in photo-injectors, AIP Conference Proceedings 581 (2001) 87-106.

[44] R. Fowler, L. Nordheim, Electron emission in intense electric fields, Proceedings of the Royal Society of London A: Mathematical, Physical and Engineering Sciences 119 (1928) 173-181.

[45] A. Bret, L. Gremillet, M. E. Dieckmann, Multidimensional electron beam-plasma instabilities in the relativistic regime, Physics of Plasmas 17 (2010) 120501.

[46] A. B. Mikhailovskii, Theory of plasma instabilities, Vol 1: instabilities of a homogeneous plasma, Springer, 1974.

[47] S. P. Gary, Theory of Space Plasma Microinstabilities, Cambridge University Press, 1993.

[48] A. M. Bykov, A. Brandenburg, M. A. Malkov, S. M. Osipov, Microphysics of cosmic ray driven plasma instabilities, Space Science Reviews 353 (2013) 125-156.

[49] R. C. Davidson, C. S. Wu, Ordinarymode electromagnetic instability in high plasmas, Physics of Fluids 13 (1970) 1407.

[50] R. C. Tautz, J.-I. Sakai, Magnetic field amplification in anisotropic counterstreaming pair plasmas, Physics of Plasmas 14 (2007) 012104.

[51] B. N. Breizman, in: B. B. Kadomtsev (Ed.), Reviews of plasma physics Vol.15, Consultants Bureau, New York, 1990.
[52] L. Sironi, D. Giannios, Relativistic pair beams from tev blazars: A source of reprocessed gev emission rather than intergalactic heating, The Astrophysical Journal 787 (2014) 49.

[53] I. Rafighi, S. Vafin, M. Pohl, J. Niemiec, Plasma effects on relativistic pair beams from tev blazars: Pic simulations and analytical predictions, Astronomy \& Astrophysics 607 (2017) A112.

[54] R. Schlickeiser, Relativistic kinetic theory of plasma waves, Physica Scripta T75 (1998) 33.

[55] M. Lazar, R. Schlickeiser, Relativistic kinetic dispersion theory of linear parallel waves in magnetized plasmas with isotropic thermal distributions, New Journal of Physics 8 (2006) 66.

[56] B. D. Fried, S. D. Conte, The plasma dispersion function, Academic Press, New York, 1961.

[57] D. Ibscher, M. Lazar, M. J. Michno, R. Schlickeiser, Towards a complete parametrization of the ordinary-mode electromagnetic instability in counterstreaming plasmas. i. minimizing ion dynamics, Physics of Plasmas 20 (2013) 012103.

[58] P. Chang, A. E. Broderick, C. Pfrommer, E. Puchwein, A. Lamberts, M. Shalaby, The effect of nonlinear landau damping on ultrarelativistic beam plasma instabilities, The Astrophysical Journal 797 (2014) 110.

[59] S. Y. Mironov, A. V. Andrianov, E. I. Gacheva, V. V. Zelenogorsky, A. K. Potemkin, E. A. Khazanov, P. Boonpornprasert, M. Gross, J. D. Good, I. Isaev, D. Kalantaryan, T. Kozak, M. Krasilnikov, H. Qian, X. Li, O. Lishilin, D. Melkumyan, A. Oppelt, Y. Renier, T. Rublack, M. Felber, H. Huck, Y. L. Chen, F. Stephan, Spatio-temporal shaping of photocathode laser pulses for linear electron accelerators, Phys. Usp. 60 (2017) $1039-1050$

[60] J. Good, A. Andrianov, G. Asova, P. Boonpornprasert, Y. Chen, E. Gacheva, et al., Preliminary On-Table and Photoelectron Results from the PITZ Quasi-Ellipsoidal Photocathode Laser System, in: Proceedings of International Free Electron Laser Conference (FEL'17), International Free Electron Laser Conference, JACoW, Geneva, Switzerland, 2018, pp. 426-428.

[61] R. C. Davidson, Kinetic waves and instabilities in a uniform plasma, in: M. N. Rosenbluth, R. Z. Sagdeev (Eds.), Basic plasma physics: selected chapters, Elsevier, 1989.

[62] R. Schlickeiser, Cosmic ray astrophysics, Springer, 2002

[63] R. Schlickeiser, P. H. Yoon, On the marginal instability threshold condition of the aperiodic ordinary mode, Physics of Plasmas 21 (2014) 072119 . 